1 Growth Factor Independence (GFI) 1B-mediated transcriptional repression and 2 lineage allocation require Lysine Specific Demethylase (LSD)1-dependent recruitment of the $\mathrm{BHC}$ complex

5 David McClellana, Mattie J. Casey ${ }^{a}$, Diana Bareyan ${ }^{a}$, Helena Lucente ${ }^{a}$, Christopher Ours ${ }^{b}$, 6 Matthew Velinder ${ }^{c}$, Jason Singer ${ }^{c}$, Mehraju Din Lone ${ }^{a}$, Wenxiang Sun ${ }^{a}$, Yunuen Coria ${ }^{a}$, Clinton

9 a Department of Oncological Sciences, University of Utah School of Medicine, Salt Lake City, 10 UT, U.S.A.

11 b Department of Pediatrics, University of Utah School of Medicine, Salt Lake City, UT, U.S.A.

12 Primary Children's Hospital, Salt Lake City, UT, U.S.A.

13 c Department of Genetics, University of Utah School of Medicine, Salt Lake City, UT, U.S.A.

14 d Center for Investigational Therapeutics, Huntsman Cancer Institute, Salt Lake City, UT, U.S.A.

15 e Nuclear Control of Cell Growth and Differentiation Program, Huntsman Cancer Institute, Salt 16 Lake City, UT, U.S.A.

\# Corresponding author: MEE2MJ@hscmail.mcc.virginia.edu

Materials and Methods: 2,128 words

21 Introduction, Results, Discussion: 4,405 words

\title{
23 Keywords
}

24 GFI1B, LSD1, BHC Complex, HMG20A, HMG20B, GSE1, Transcriptional Repression,

25 SNAG domain, Erythropoiesis, Hematopoiesis

\section{Running Title}

28 GFI1B function requires BHC complex binding via LSD1 


\section{ABSTRACT}

30 Growth Factor Independence (GFI)1B coordinates assembly of transcriptional repressor

31 complexes comprised of co-repressors and histone modifying enzymes to control gene

32 expression programs governing lineage allocation in hematopoiesis. Enforced expression

33 of GFI1B in K562 erythroleukemia cells favors erythroid over megakaryocytic

34 differentiation, providing a platform to define molecular determinants of binary fate

35 decisions triggered by GFI1B. We deployed proteome-wide proximity labeling to identify

36 factors whose inclusion in GFI1B complexes depends upon GFI1B's obligate effector,

37 Lysine Specific Demethylase (LSD)1. We show that GFI1B preferentially recruits core

38 and putative elements of the BRAF-histone deacetylase (HDAC) (BHC) chromatin

39 remodeling complex (LSD1, RCOR1, HMG20A, HMG20B, HDAC1, HDAC2, PHF21A,

40 GSE1, ZMYM2 and ZNF217) in an LSD1-dependent manner to control erythroid fate

41 specification. Among these, depletion of both HMG20A and HMG20B, or GSE1 block

42 GFI1B-mediated erythroid differentiation, phenocopying impaired differentiation brought

43 on by LSD1 depletion or disruption of GFI1B - LSD1 binding. These findings demonstrate

44 the central role of the GFI1B_LSD1 interaction as a determinant of BHC complex

45 recruitment to enable cell fate decisions driven by GFI1B. 


\section{INTRODUCTION}

47 Transcriptional regulation is critical for cell growth, homeostasis, and fate determination

48 in multicellular organisms. In hematopoiesis, cell surface receptors, signaling networks,

49 transcription factors and their effectors cooperate to influence chromatin state and gene

50 expression, directing hematopoietic progenitors toward specific fates, and once achieved,

51 ensuring proper function for each cell type. Failure to properly control chromatin structural

52 dynamics governing stage-specific gene expression programs can cause qualitative and

53 quantitative hematopoietic disorders, including malignancies. Failures can arise from

54 altered expression of transcription factors or from malfunction or misdirection of their

55 partners (1-3). Thus, knowing the composition of protein complexes that regulate gene

56 expression by cell fate specifying transcription factors is essential to understanding

57 normal and malignant hematopoiesis, and may have therapeutic relevance (4).

Growth Factor Independence (GFI) family transcriptional repressors, GFI1 and

59 GFI1B, are master regulators of developmental hematopoiesis $(5,6)$. GFI1 controls

60 hematopoietic stem cell self-renewal, B- and T-lymphopoiesis, and directs the

61 granulocyte versus monocyte fate decision during late stages of myeloid differentiation

62 (7-9). Notably, Gfi1-null mice are viable, but are characterized by severe neutropenia with

63 compensatory monocytosis and sensorineural hearing loss (10-12). Altered GFI1

64 expression is found in multiple hematologic malignancies, most notably acute myeloid

65 leukemia (AML) and T-cell acute lymphoblastic leukemia (T-ALL) (13-17). Moreover, loss

66 of function mutations in GFI1 cause severe congenital neutropenia (SCN) type 2 and

67 nonimmune chronic idiopathic neutropenia (CIN) of adults, both of which predispose to

68 AML $(18,19)$. GFI1B is required for definitive erythropoiesis and megakaryopoiesis, and 
69 like GFI1, also helps to maintain hematopoietic stem cell quiescence (20-23). In mice,

70 Gfi1b nullizygosity is lethal at embryonic day (E) 15 due to erythropoietic failure (24).

71 Mutations in GFI1B are associated with both qualitative and quantitative disorders of

72 platelets and elevated GFI1B expression is found in AML (25-28). However, the role of

73 GFI1B in AML is uncertain. Despite GFI1B often being increased in AML patients (29-

74 32), a recent study suggests low GFI1B expression correlates with poor patient outcome

75 and that in mice, Gfi1b depletion accelerates progression to AML from myelodysplasia

76 driven by a NUP98-HOXD13 fusion protein (33). Collectively, these observations suggest

77 widespread contributions by GFI family proteins to lineage allocation during normal

78 hematopoiesis and both oncogenic and oncosuppressor roles in hematologic

79 malignancies. A clear understanding of GFI1/1B contributions to hematopoiesis and

80 malignancy requires comprehensive accounting of their protein partners and their

81 dependency relationships during cell fate decision making.

82 GFI family proteins feature a shared structural organization (5, 34). Both GFI1 and

83 GFI1B are comprised of a Snail/Slug/Gfi1 (SNAG) domain and a highly conserved

84 concatemer of six $\mathrm{C}_{2} \mathrm{H}_{2}$-type zinc-fingers $(\mathrm{ZnF})$ at their $\mathrm{N}$ - and $\mathrm{C}$-termini, respectively.

85 Linker regions with limited conservation separate the SNAG and ZnF domains.

86 Sequence-specific DNA binding by both GFI1 and GFI1B is coordinated by ZnFs 3, 4 and

$875(32,35-37)$, while ZnFs 1,2 , and 6 enable protein-protein interactions with an

88 incompletely defined subset of transcriptional partners (38-41). Linker regions provide a

89 platform for protein binding and post-translational modifications that regulate innate

90 functions of GFI proteins (40, 42). GFI1 and GFI1B SNAG domains differ only by a

91 conservative $S \rightarrow T$ substitution at amino acid 14, but are otherwise identical. Moreover, 
92 the SNAG domain is near invariant among orthologs from human to zebrafish,

93 underscoring the critical role of the SNAG domain and its binding partners. Prominent

94 among these is Lysine Specific Demethylase (LSD)1 (KDM1A, AOF2, BHC110, NPAO,

95 KIAA0601), which is required for transcriptional repression by GFI proteins (10, 43). The

96 SNAG domain is necessary and sufficient for LSD1 binding by GFI1 and GFI1B (44).

97 SNAG domain deletion, alanine substitution at proline 2 (P2A) or leucine substitution at

98 lysine 8 (K8L) of the SNAG domain abolishes LSD1 binding to GFI family proteins and

99 profoundly impairs their activity in multiple functional assays (43, 45-47).

100

LSD1 is a flavin-dependent monoamine oxidase that demethylates mono- and di-

101 methylated histone 3 lysines 4 and $9(\mathrm{H} 3 \mathrm{~K} 4 \mathrm{me} 1 / 2$ and H3K9me1/2) to impact chromatin

102 structure and gene expression (48, 49). LSD1 also demethylates non-histone targets

103 including p53, STAT3, ER $\alpha$, MYPT1, HIF1 $\alpha$, DNMT1, E2F and SOX2, suggesting broad

104 influence over cellular homeostasis (50-57). LSD1 operates within multiple assemblies

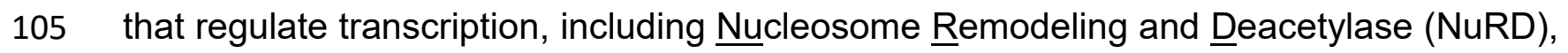

106 C-terminal Binding Protein (CtBP), Mixed Lineage Leukemia (MLL) coactivator and

107 B $R A F-\underline{H i s t o n e}$ deacetylase $(\mathrm{BHC})$ complexes (58-62). Its inclusion in distinct multiprotein

108 assemblies suggests both enzymatic and structural contributions to transcriptional

109 control, yet affiliations between LSD1-containing complexes and the DNA binding

110 proteins that recruit them are poorly understood.

111 We leveraged GFI1B-driven erythroid differentiation in K562 cells, GFI1B fusion

112 proteins with a promiscuous biotin ligase $\left(\operatorname{Bir}^{*}\right)(63,64)$ and unbiased mass-spectral

113 quantitation to define proximity relationships between GFI1B and partners that depend 114 upon GFI1B - LSD1 binding. We show that GFI1B - LSD1 binding is necessary but not 
115 sufficient for GFI1B-mediated erythroid differentiation in K562 cells. We also show that

116 recruitment of core (65) (LSD1, RCOR1, HDAC1, HDAC2, HMG20B and PHF21A) and

117 putative $(66,67)$ (HMG20A, GSE1, ZMYM2, and ZNF217) BHC complex components by

118 GFI1B depends upon GFI1B_LSD1 binding and that depletion of candidate BHC 119 complex proteins HMG20A and HMG20B, as well as GSE1 impairs GFI1B-mediated 120 erythroid differentiation even though GFI1B - LSD1 interaction potential remains. Our

121 results show that GFI1B-mediated lineage allocation depends upon LSD1's function as a

122 scaffolding component for BHC complex assembly and highlight the need to consider

123 protein assemblies as modules which act in concert as determinants of cell fate.

125 RESULTS

126 LSD1 depletion impairs GFI1B-mediated erythroid differentiation

127 The human erythroleukemia cell line, K562, displays erythroid differentiation with 128 enforced GFI1B expression (68), reflected by hemoglobinization and benzidine positive 129 staining (Fig. 1A). Likewise, K562 cells adopt megakaryocytic features, including CD61 130 expression, with tetradecanoyl-1-phorbol-13-acetate (TPA) treatment (Fig. 1B). These

131 dual fates are mutually exclusive, enabling GFI1B dependency relationships relevant to

132 fate specification to be discerned. Notably, deletion of the GFI1B SNAG domain or 133 placement of an epitope tag at the GFI1B N-terminus abolishes GFI1B-driven erythroid 134 differentiation, suggesting primacy of the SNAG domain for this phenotype (68-70). Being 135 necessary and sufficient for LSD1 binding, we surmised that disrupting or blocking access 136 to the SNAG domain would impair LSD1 recruitment, and by extension, that LSD1 137 function would be required for GFI1B-driven erythroid differentiation. To confirm this, we 
138 depleted K562 cells of LSD1 and tested GFI1B's ability to induce erythroid differentiation

139 and for TPA to trigger cell surface expression of CD61. Immunoblots show depletion of

140 LSD1 by two distinct shRNA constructs relative to a scramble control sequence, and

141 equivalent expression of GFI1B (Fig. 1C). LSD1 depletion almost completely eliminates

142 the detection of benzidine positive cells in response to enforced expression of GFI1B

143 compared to controls yet had limited impact on CD61 expression at the cell surface

144 following TPA treatment (Fig. 1D and E). LSD1 depletion also blocked expression of alpha

$145(H B A 1 / 2)$ and beta $(H B B)$ globins and alpha hemoglobin stabilizing protein $(A H S P)$, as

146 well as 5-aminolevulinic acid synthase $(A L A S) 2$ and ferrochelatase $(F E C H)$, which are

147 rate limiting enzymes required for heme biosynthesis. Expression of the erythroid fate

148 marker glycophorin A (GYPA) was also significantly impaired (Fig. 1F and G). To confirm

149 contributions to GFI1B-mediated transcriptional repression, we examined mRNA levels

150 for GFI1B direct targets CDKN1A, GFI1B and MYB in the context of LSD1 depletion.

151 Repression of each gene was reversed by LSD1 depletion (Fig. 1H). Together these data

152 show that LSD1 enables transcriptional repression by GFI1B to trigger an erythroid fate

153 specifying program in K562 cells and that LSD1 is dispensable for TPA-driven 154 megakaryocytic differentiation.

\section{GFI1B_LSD1 binding is required for GFI1B-mediated erythroid differentiation}

LSD1 cooperates with an extensive roster of DNA binding proteins to influence

158 gene expression (71), and therefore could influence this binary fate decision through

159 partnerships with factors other than GFI1B. To test whether an interaction between GFI1B

160 and LSD1 is needed for GFI1B-mediated erythroid differentiation, we compared K562 
161 cells with enforced expression of GFI1B-WT or its -P2A derivative. The P2A substitution

162 abolishes GFI1B_LSD1 binding, as well as transcriptional repression by fusion proteins

163 between Gal4 and either GFI1B or the SNAG domain in isolation (Fig. 2A and B). While

164 GFI1B-WT triggers an erythroid differentiation program in K562 cells, as reflected by 165 hemoglobinization and erythroid-specific gene expression, GFI1B-P2A fails to do so (Fig.

166 2C-F). GFI1B-WT expression also blocks TPA-induced megakaryocytic differentiation in

167 K562 cells, yet GFI1B-P2A displays no such antagonism (Fig. 2G). Additionally, failure

168 by GFI1B-P2A either to trigger erythroid differentiation or to block CD61 expression in

169 response to TPA correlates with impaired transcriptional repression of direct GFI1B

170 targets (Fig. 2H). These findings indicate LSD1 recruitment by GFI1B is necessary to

171 direct the erythroid fate-specifying program and is not required for TPA-induced

172 megakaryocytic differentiation.

173

174 GFI1B partners identified by proximity-dependent biotinylation

175 GFI1B_LSD1 binding is required for GFI1B-mediated transcriptional control and

176 fate specifying functions in hematopoiesis $(43,45)$. However, LSD1 is a constituent of

177 multiple transcriptional regulatory complexes. Which among these governs GFI1B

178 function to direct erythroid differentiation is unknown. To more comprehensively

179 catalogue GFI1B partners, we engineered a chimeric protein, GFI1B-BirA*:HA,

180 comprised of the complete GFI1B primary structure fused in frame with a promiscuous

181 variant of the biotin ligase BirA (BirA*) from Aquifex aeolicus $(63,64,72)$ and a C-terminal

182 HA epitope tag under the control of a doxycycline-inducible promoter. Proteins with a

183 proximity relationship to BirA*:HA or GFI1B-BirA*:HA are biotinylated spontaneously and 
184 can be further characterized (Fig. 3A). GFI1B-BirA*:HA or BirA*:HA were inducibly

185 expressed in K562 cells and proteome-wide biotinylation was compared to K562 cells

186 transduced with vector only. GFI1B-BirA*:HA and BirA*:HA were equivalently expressed

187 and produced a broad array of biotinylated protein substrates as reflected by streptavidin

188 (SAv):HRP-mediated detection in whole cell extracts (Fig. 3B). Both qualitative and

189 quantitative differences in the pattern of biotinylated proteins are apparent, suggesting

190 preferential labeling of targets by BirA*:HA when anchored to GFI1B. In the vector control,

191 a single 72kD band was seen, likely representing propionyl-CoA carboxylase expression

192 in K562 cells (73). To further validate the proximity labeling technique, we confirmed

193 enrichment for established GFI1B associated proteins, LSD1 and RCOR1, among

194 biotinylated proteins from cells expressing GFI1B-BirA*:HA relative to those expressing

195 non-targeted BirA*:HA. LSD1 binds directly to the GFI1/1B SNAG domain while RCOR1

196 engages GFI1B through LSD1 (43). LSD1 and RCOR1 were readily identified among

197 biotinylated proteins from GFI1B-BirA*:HA-expressing cells collected on SAv-Sepharose

198 beads, but only minimally so from cells expressing BirA*:HA or empty vector (Fig. 3C).

199 Serving as an internal control for equal target biotinylation potential, GFI1B-BirA*:HA and

200 BirA*:HA were comparably modified with biotin, purified on SAv-Sepharose and detected

201 by $\alpha-\mathrm{HA}$ western blot (Fig. 3C). These findings indicate preferential biotinylation of factors

202 known to have a proximity relationship with GFI1B. Moreover, they confirm detection of

$2031^{\circ}$ (intrinsic), $2^{\circ}$ (direct binding) and $3^{\circ}$ (indirect binding) labeling events and validate this

204 platform for identifying GFI1B partners proteome-wide.

205 To comprehensively catalog GFI1B proximity partners, biotinylated proteins were 206 collected and purified on SAv-Sepharose beads from whole cell extracts of K562 cells 
207 inducibly expressing BirA*:HA or GFI1B-BirA*:HA. Assays were performed in triplicate.

208 Purified proteins were denatured, fractionated over acrylamide gels, excised, subjected

209 to trypsin digestion in situ, then analyzed by LC-MS-MS to unequivocally establish their

210 identities and extrapolate their abundance from sum read intensities (Supplementary

211 Data S1). Fold enrichment (GFI1B-BirA*:HA vs. BirA*:HA only) was determined using

212 average sum read intensities from triplicate analysis for proteins purified from GFI1B-

213 BirA*:HA-expressing cells compared to those expressing BirA*:HA only. P-values were

214 determined with a two-tailed Student's $t$-test using variances of the 20 closest ranked

215 proteins to account for differences in protein abundance across the data set. Fold

216 enrichment ( $x$-axis) for each protein is shown relative to $p$-value ( $y$-axis) in a volcano plot

217 with logarithmic axes, and statistically significant outliers are labeled (on the plot, and

218 inset) (Fig. 3D). Notably, we observed hemoglobinization of cells expressing GFI1B-

219 BirA*:HA comparable to those expressing GFI1B only, but not for cells expressing 220 BirA*:HA (data not shown).

221 GFI1B is best known as a transcriptional repressor, and its contributions to multiple

222 functional assays can be traced to this role $(20,74-79)$. Therefore, we focused first on

223 partnerships between GFI1B and determinants of chromatin structure and transcriptional

224 control. In the GFI1B-BirA*:HA proximitome, we identified elements of multiple complexes

225 highly relevant to regulated gene expression (Fig. 4). Components of protein complexes

226 enriched in the GFI1B-BirA*:HA proximitome relative to BirA*:HA control are shaded

227 black. Those present, but not significantly above control are shaded gray and those not

228 found in the GFI1B-BirA*:HA proximitome are shown on white background. Prominent

229 among those proteins present and enriched in the GFI1B-BirA*:HA proximitome are 
230 members of the BHC, CtBP and BAF complexes, from which we discovered 10/10 (core

$231+$ putative), $7 / 8$ and 13/15 complex members, respectively. Similarly, we observed each

232 component of the NuRD complex in the GFI1B-BirA*:HA proximitome, yet only a subset

233 of these was found enriched relative to the BirA*:HA control. Components from several

234 other complexes (NuRF, CHRAC, canonical and non-canonical PRC1, PRC2, SIN3A and

$235 \mathrm{NCoR}$ ) were found, and for each, some elements enriched, suggesting they also

236 contribute to GFI1B-mediated transcriptional control and lineage allocation. Noteworthy

237 is identification of GFI1B, being physically tethered to BirA* as the most highly enriched

238 protein found in this unbiased screen, further validating the strategy from a quantitative

239 perspective.

241 Identification of LSD1-dependent GFI1B binding partners

242 LSD1 binding is necessary for GFI1B function (10, 43). However, discovering 243 elements from functionally distinct transcriptional regulatory complexes within the GFI1B

244 proximitome suggests an important interplay between these elements and LSD1.

245 Moreover, at least three of these complexes (BHC, NuRD and CtBP) contain LSD1 as a

246 core component $(58,60,80)$. Given the importance of LSD1 for GFI1B function, we

247 hypothesized one or more transcriptional regulatory complexes represented in the GFI1B

248 proximitome would be recruited in an LSD1-dependent manner, and if its components

249 were depleted, would impair GFI1B function even when GFI1B_LSD1 binding potential

250 is preserved. To address this hypothesis, we engineered expression constructs encoding

251 fusion proteins comprised of BirA* and either LSD1 binding-competent forms of GFI1B

252 (GFI1B-BirA*:HA and SNAG-BirA*:HA) or GFI1B variants with impaired LSD1 binding 
253 (GFI1B-P2A-BirA*:HA and GFI1B- $\triangle$ SNAG-BirA*:HA). A BirA*:HA construct served as an

254 additional negative control (Fig. 5A). Fusion proteins were inducibly expressed in K562

255 cells and their biotinylating ability compared in whole cell lysates by SDS-PAGE,

256 transblotting and SAv:HRP detection (Fig. 5B). Equivalent expression and robust,

257 proteome-wide biotinylation were observed for each construct induced with doxycycline.

258 We then tested the ability of each fusion protein to establish proximity relationships with

259 known SNAG domain binding proteins, LSD1 and RCOR1 $(43,45)$. As expected, GFI1B-

260 BirA*:HA and SNAG-BirA*:HA display clear enrichment for biotinylated LSD1 and RCOR1

261 in SAv-Sepharose pull downs, while LSD1 non-binding variants (P2A and $\triangle$ SNAG) show

262 biotinylation comparable to background seen for the BirA*:HA negative control (Fig. 5C).

263 Equally important, we observed BirA*:HA biotinylation comparable to each of its fusion

264 proteins, suggesting equal capacity for spontaneous biotinylation and mitigating concerns

265 that the fusion partner might adversely impact BirA* enzyme activity. Collectively, these

266 results support use of GFI1B variants to unambiguously define the LSD1-dependent

267 elements of GFI1B complexes governing transcriptional control and cell fate specification.

268 In pursuit of this goal, we deployed these tools for quantitative proximitome

269 analyses to compare partnerships between GFI1B forms which are either capable of

270 (GFI1B-BirA*:HA and SNAG-BirA*:HA), or deficient in (GFI1B-P2A-BirA*:HA, GFI1B-

$271 \Delta$ SNAG-BirA*:HA and BirA*:HA) LSD1 binding. Fusion protein expression was induced

272 by addition of doxycycline and biotinylated proteins collected on SAv-Sepharose beads

273 as above. After high stringency washing, affinity purified proteins were analyzed by LC-

274 MS-MS as described above. Triplicate read intensities were used to calculate an average

275 sum read intensity for each protein identified in the mass spectral screen. Proteins were 
276 ranked according to average sum read intensities in the LSD1 non-binding control and

277 fold enrichment for each protein was calculated as a ratio of average sum read intensities

278 between LSD1 binding and non-binding variants. $P$-values were calculated from variation

279 among the twenty nearest ranked neighbors for each protein in the data set. Compared

280 to LSD1 non-binding variants GFI1B- $\triangle$ SNAG-BirA*:HA (Fig. 6A) and GFI1B-P2A-

281 BirA*:HA (Fig. 6B), we observed enrichment for nine of the ten core (LSD1, RCOR1,

282 PHF21A, HMG20B, HDAC1and HDAC2) and putative (GSE1, HMG20A, and ZMYM2)

283 components of the BHC complex in the GFI1B-BirA*:HA proximitome. These results

284 indicate BHC complex recruitment is lost with impaired GFI1B_LSD1 binding. To focus

285 specifically on the SNAG-LSD1 interaction as a determinant of BHC complex

286 recruitment, we determined enrichment for BHC complex components in the SNAG-

287 BirA*:HA proximitome relative to BirA*:HA only. Again, we find enrichment for these nine

288 BHC complex components in the SNAG-BirA*:HA proximitome.

289 LSD1, HDAC1, and HDAC2 are shared among the BHC, CtBP and NuRD

290 complexes, while RCOR1 is found in both BHC and CtBP complexes. To confirm the

291 LSD1-dependence of BHC complex recruitment specifically, we surveyed the top 100

292 enriched proteins in each binary proximitome comparison (GFI1B-BirA*:HA vs. GFI1B-

$293 \Delta$ SNAG-BirA*:HA, GFI1B-BirA*:HA vs. GFI1B-P2A-BirA*:HA, and SNAG-BirA*:HA vs.

294 BirA*:HA) for the unique components of BHC, CtBP and NuRD complexes (Table 1 and

295 Table S1). While unique components of the BHC complex were found in each binary

296 proximitome comparison performed, none of those unique to the CtBP or NuRD

297 complexes were observed. These findings indicate that among LSD1-containing 
298 complexes detected in the GFI1B proximitome, only the BHC complex displays LSD1-

299 dependence.

300

301 GFI1B-mediated transcriptional repression and cell fate require the BHC complex

To confirm findings from mass spectral screens, we first assessed associations

303 between GFI1B and candidate BHC complex components and tested their dependence

304 upon GFI1B_LSD1 binding. We focused on unique elements of the BHC complex.

305 GFI1B-BirA*:HA, GFI1B-P2A-BirA*:HA, GFI1B- $\triangle$ SNAG-BirA*:HA or SNAG-BirA*:HA

306 were inducibly expressed in K562 cells and biotinylated proteins purified on SAv-

307 Sepharose beads. HMG20A, HMG20B, and PHF21A were then quantified among purified

308 proteins by western blot. Each was enriched among proteins purified from cells

309 expressing GFI1B-BirA* fusions capable of LSD1 binding relative to non-binding controls

310 (Fig. 7A). Notably, no such enrichment was observed for SMARCB1 or SMARCC1,

311 members of the SWI/SNF complex that showed enrichment but no LSD1 dependence in

312 GFI1B proximitome analyses. Nor did SMARCB1 or SMARCC1 show a proximity

313 relationship when SNAG-BirA*:HA was employed, confirming a distinct and LSD1-

314 independent means of associating with GFI1B. Using flag-tagged wild type and -P2A

315 forms of GFI1B, we extended findings from proximity labeling studies to traditional co-

316 precipitation assays. For HMG20A, HMG20B, and PHF21A, binding to GFI1B-P2A was

317 significantly impaired relative to wild type GFI1B, confirming dependence upon LSD1

318 recruitment (Fig. 7B-D). This observation is further supported by co-precipitation assays

319 showing interactions with LSD1 for both HMG20A and HMG20B (Fig. 7E). 
LSD1 is required for both GFI1B function and BHC complex recruitment, but

321 whether LSD1 exerts its impact through BHC complex recruitment is not known. To

322 address this question, we targeted unique BHC complex components for depletion in

323 K562 cells then tested GFI1B's ability to direct changes in cell fate and gene expression

324 (Fig. 8). HMG20A and HMG20B are known to bind structured elements in DNA in a

325 sequence non-specific manner (81-83). They have been shown to have an oppositional

326 role in neuronal differentiation via modulation of REST-responsive genes $(80,84)$. Their

327 contributions to hematopoietic differentiation have not been described. HMG20A and

328 HMG20B were depleted alone or together using inducible shRNA constructs (Fig. 8A),

329 then effects of enforced GFI1B expression on K562 cell differentiation and gene

330 expression were assessed. While neither HMG20A nor HMG20B depletion alone had a

331 notable impact on K562 cell hemoglobinization, simultaneous depletion of both factors

332 prevented this GFI1B-driven phenotype (Fig. 8B). Likewise, impaired TPA-induced

333 megakaryocytic differentiation brought on by enforced GFI1B expression was not

334 significantly affected by depletion of either HMG20A or HMG20B alone. Yet, their

335 concurrent depletion enabled accumulation of CD61 on the K562 cell surface despite

336 GFI1B overexpression (Fig. 8C). Erythroid differentiation blockade brought on by

337 concurrent depletion of HMG20A and HMG20B correlated with reduced expression of

338 genes required for heme and globin chain synthesis and with loss of repression of GFI1B

339 target genes (Fig. 8D-F). Notably, LSD1 expression is unaffected by HMG20A or

340 HMG20B depletion (Fig. 8A). Thus, HMG20A and HMG20B make functional contributions

341 to LSD1-dependent outcomes driven by GFI1B. Their concurrent absence impairs GFI1B-

342 driven erythroid fate in K562 cells and enables alternate, TPA-driven megakaryocytic 
343 differentiation in the context of enforced GFI1B expression that would normally block it.

344 Moreover, unlike the oppositional relationship between HMG20A and HMG20B observed

345 in REST-regulated neural differentiation, these findings intimate functional redundancy

346 between them in GFI1B-mediated transcription and its control of cellular identity.

347 To extend observations made with HMG20A and HMG20B, we tested the effects

348 of GSE1 depletion on GFI1B functions in transcriptional repression and erythroid fate 349 specification in K562 cells. GSE1 is a proline-rich coiled-coil domain protein implicated in 350 pathogenesis of breast and gastric cancers $(85,86)$. It is widely expressed in human cells,

351 but its role in cell fate decisions in hematopoiesis have not been studied. As for HMG20

352 proteins, reduced GSE1 expression blocks erythroid differentiation and gene expression

353 changes in K562 cells which depend upon the GFI1B_LSD1 binding relationship (Fig.

354 9). Together with results from LSD1, HMG20A and HMG20B depletion, these data

355 highlight that LSD1 is necessary but not sufficient for GFI1B-driven outcomes and

356 showcase a critical role for the BHC complex as an LSD1-dependent determinant of

357 GFI1B-mediated transcriptional repression and cell fate decisions in hematopoiesis.

\section{DISCUSSION}

The control of gene expression involves DNA binding proteins that recognize

361 discrete sequence elements in regulatory regions of genes, directing site-selective

362 assembly of multiprotein complexes that modulate chromatin structure, and ultimately,

363 access to coding regions by the basal transcription machinery $(87,88)$. By deploying

364 individual elements in a modular fashion, cells insure flexibility and economy as they 365 orchestrate transcriptional programs to control the specification of cell fate. Proper 
366 function of these complexes is layered upon a spatial and temporal order of events that

367 combine to create a flow of information needed for a specific outcome among the myriad

368 of possibilities. Yet, this strategy necessarily creates dependency relationships between

369 factors cooperating to accomplish functions required for an outcome to be realized.

370 Malfunction of just one complex component, or disordered operation of a complex in

371 space or time may impair functional outputs and thus mask the underlying molecular

372 complexities required to achieve them.

373 The relationship between GFI family proteins and LSD1 exemplifies this theme.

374 Both GFI1 and GFI1B require LSD1 binding for transcriptional repression and point

375 mutations in their SNAG domains completely abolish activity in multiple functional assays

$376(43,45,46,89)$. It is not, however, immediately apparent that other factors might enable

377 LSD1's central role, and identifying these collaborators is technically challenging. We

378 deployed an unbiased, proteome-wide proximity labeling strategy based upon a

379 promiscuous variant of BirA $\left(\operatorname{Bir}^{*}\right)$ to prospectively and systematically label with biotin

380 factors that dwell in the vicinity of GFI1B and then to determine functionally relevant

381 partners which are recruited in a manner requiring LSD1, its obligate effector. In so doing,

382 we show that while LSD1 recruitment is critical to GFI1B-driven erythroid differentiation,

383 it does not operate alone as its singular depletion in K562 cells may suggest. Rather,

384 LSD1 is responsible for selective recruitment of the BHC complex, implying that its

385 contributions to GFI1B-mediated outcomes may reflect both its intrinsic demethylase

386 activity and its role as a scaffolding component for this multiprotein complex. A model

387 summarizing the collaborative relationship between LSD1, BHC complex components

388 and other partners in GFI1/1B-mediated transcriptional repression is shown (Fig. 10). 
LSD1 displays in vitro demethylase activity toward peptides containing mono- and di-methylated lysines $(\mathrm{K})$, best exemplified by demethylation of H3K4me1/me2, and

391 toward purified histones $(48,90)$. However, in a chromatinized context, methylated

392 histones are poor substrates for LSD1 (91). Efficient demethylation of chromatinized 393 histones by LSD1 requires concurrent RCOR1 binding, and this activity can be further 394 stimulated by an interaction between RCOR1 and SUMO2/3 (91, 92). Likewise, LSD1's 395 demethylase activity is favored by HDAC1-mediated deacetylation of LSD1-K374ac (93).

396 Observations such as these support the notion that LSD1's demethylase activity may

397 hinge upon its inclusion in multiprotein complexes to enable its proper post-translational 398 modifications and allosteric modulation. Additionally, by serving as a scaffold, LSD1 may 399 enable regulation of other components of the BHC complex, or perhaps discrete 400 regulatory modules recruited to operationalize GFI1B activity as a transcriptional

401 repressor. Absent these cooperative relationships, LSD1 may fail to execute its obligate 402 effector role for GFI1B despite the means of its recruitment by GFI1B being intact. 403 Investigating these possibilities presents a daunting challenge, but one that can now be 404 tackled using the situational capabilities of proximity labeling approaches. Moreover, a 405 holistic view of LSD1 contributions to GFI1B's repressor and cell fate specifying activities 406 is consistent with both its pivotal role and its dependence upon partnerships.

407 To establish evidence supporting this view, we focused on BHC complex 408 components HMG20A and HMG20B as LSD1 partners. This focus was stimulated by 409 studies in Schizosaccharomyces pombe concerning the role of Lsd1/2 in replication fork 410 pausing associated with mating type class switching (94). Lsd1/2 is required for growth 411 and viability in S. pombe. Notably, S. pombe strains rendered catalytically inactive at /sd1/ 
412 Isd2 are viable and do not phenocopy Isd1/Isd2 deletion, suggesting they have a non-

413 enzymatic role. Moreover, Lsd1 contains a C-terminal HMG domain that is lacking in

414 mammalian LSD1, and HMG domain mutations impair mating type class switching

415 through defective imprinting at the mat1 locus brought on by dysfunctional replication fork

416 pausing $(48,94)$. The importance of the Lsd1 HMG domain to a phenotype dependent

417 upon structural change in DNA, the absence of the HMG domain in mammalian LSD1,

418 the suggestion of a non-enzymatic role for Lsd1 and the inclusion of HMG20 proteins in

419 the GFI1B proximitome made HMG20A and HMG20B attractive candidates in GFI1B-

420 LSD1- dependent outputs in K562 cells. Our finding that concurrent HMG20A/B depletion

421 blocks LSD1-dependent functions of GFI1B suggests that their inclusion in the BHC

422 complex is essential to enable LSD1 actions. Similar observations made by depleting

423 GSE1 reinforce this notion. Notably, HMG20A and HMG20B are ubiquitously expressed

424 (95). They contain N-terminal HMG boxes which bind DNA in a sequence non-specific

425 manner and C-terminal coiled-coil domains required for dimerization and incorporation

426 into multiprotein complexes $(67,84)$. In neural development, HMG20A and HMG20B

427 display an oppositional relationship at REST-regulated genes $(65,80,84)$. Yet, our data

428 suggest these proteins may also compensate for deficiencies in one another and that

429 perhaps the relative abundance of each may fine tune transcriptional activity of the BHC

430 complex at sites to which it has been recruited. Thus, with site-selective DNA binding

431 proteins (e.g., GFI1B), epigenetic modifiers (e.g., LSD1, HDAC1/2), their cofactors (e.g.,

432 RCOR1), and readers of epigenetic state (e.g., PHF21A) as partners, a network of

433 interdependent proteins may cooperate to direct the quality, quantity and timing of

434 transcriptional programs that govern the specification of cell fate. Our findings provide a 
435 framework for exploring the interdependent and situational relationships among these 436 classes of proteins.

\section{MATERIALS AND METHODS}

\section{Cell culture and viral transduction}

440 COS7L, HEK293T (American Type Culture Collection), MEL and 293-T-Rex-5xGal441 luciferase cells (generously provided by Raphael Margueron) were maintained in

442 Dulbecco's modified Eagle's medium (DMEM) supplemented with $10 \%$ fetal bovine serum 443 (FBS). K562 cells were maintained in Roswell Park Memorial Institute (RPMI) medium

444 supplemented with 10\% FBS and $25 \mu \mathrm{M}$ HEPES. All cell culture media was supplemented 445 with $2 \mu \mathrm{M}$ L-Glutamine, 50 units $/ \mathrm{ml}$ penicillin, and $50 \mu \mathrm{g} / \mathrm{ml}$ streptomycin. All cell media 446 and additives were obtained from Thermo-Fisher. All cell lines were verified using the

447 Geneprint 10 system (Promega). For viral production, HEK293T cells were transfected 448 with a viral vector containing the transgene along with the respective lenti- (psPAX2 and $449 \mathrm{pMD2G}$ ) or retroviral (gag/pol and VSVG) packaging plasmids using polyethylenimine 450 (PEI) $(3 \mu \mathrm{g} \mathrm{PEl}: 1 \mu \mathrm{g}$ DNA). Viral supernatant was collected 48- and 72-hours post451 transfection and clarified by filtration (Corning, $45 \mu \mathrm{m}$ ). K562 cells were transduced with 452 viral particles in the presence of $8 \mu \mathrm{g} / \mathrm{ml}$ polybrene for 16 hours. Stable cells were 453 obtained by selecting with $5 \mu \mathrm{g} / \mathrm{ml}$ puromycin for 4 days, $400 \mu \mathrm{g} / \mathrm{ml}$ G418/neomycin or 454 hygromycin for 10 days. For LSD1 and HMG20B small hairpin (shRNA) experiments, 455 K562 cells were transduced with Tet-pLKO-neo (a gift from Dmitri Wiederschain, 456 Addgene plasmid \#21916) containing either LSD1-, HMG20B-targeted shRNA or 457 scrambled control and selected in neomycin to establish stable isolates. For HMG20A 
458 and GSE1 shRNA experiments, K562 cells were transduced with EZ-Tet-pLKO-Hygro (a

459 gift from Cindy Miranti, Addgene plasmid \#85972) containing either HMG20A-, GSE1-

460 targeted shRNA or scrambled control and selected in hygromycin. To obtain double

461 HMG20A and B shRNA knockdown, K562 cells were first transduced with either HMG20B

462 or scrambled shRNA vector, selected with neomycin, and subsequently transduced with

463 either HMG20A or scrambled shRNA vector and selected with hygromycin. shRNA 464 expression was achieved by treating transduced cells with $500 \mathrm{ng} / \mathrm{ml}$ doxycycline every 46524 hours. shRNA sequences are listed in Table S2.

467 RNA extraction and gene expression analysis

468 Total RNA was prepared using RNeasy (Qiagen), as per the manufacturer's instructions, 469 including on-column DNAse I digestion to avoid potential gDNA contamination. The cDNA 470 was generated from 100-300 ng of total RNA using iScript Reverse Transcription 471 Supermix for RT-qPCR (Bio-Rad). cDNA was diluted $2 X$ with water, and $1 \mu$ of cDNA

472 was used as a template for real-time PCR (qPCR). Quantitation was performed by qPCR 473 with the CFX96 Real Time System (Bio-Rad), using $5 \mu$ SsoAdvanced Universal SYBR

474 Green Supermix (Bio-Rad), and $330 \mathrm{nM}$ of the indicated forward and reverse primers in

475 a $10 \mu$ reaction. Relative mRNA expression was determined by the $\Delta \Delta \mathrm{C}_{\mathrm{t}}$ method and 476 normalized to a GUS reference gene. Each experiment was performed in triplicate.

477 Statistical significance was determined by two-sided unpaired $t$-tests using GraphPad 478 Prism 7.0. Sequences of all oligonucleotides used are listed in Table S2. 
481 GFI1B (NM_008114.3), PHF21A (NM_008114.3), HMG20A (NM_025812.3), and

482 HMG20B (NM_010440.3) were amplified by PCR (Platinum SuperFi DNA Polymerase,

483 Invitrogen) from cDNA obtained from MEL cells and subcloned into pcDNA3.1(-)

484 (Invitrogen) with either a 3x-flag, HA, or V5 tag on the 3' end of the gene. The GFI1B-P2A

485 mutation was made by site-directed mutagenesis. Retroviral GFI1B:3x-flag vectors were

486 produced by subcloning GFI1B into the pMSCV (murine stem cell virus)-IRES-Puro vector

487 using EcoR1. GFI1B fusion proteins to Gal4 were generated by PCR amplification of the

488 GFI1B SNAG domain (amino acid 1-20) or GFI1B- $\Delta 1 C$ (amino acids 1-244 only-

489 eliminating ZnF 4, 5 and 6, which remove intrinsic DNA binding via GFI1B sequence) and

490 inserted into pcDNA3.1 N-terminally and in frame with the Gal4 DNA-binding domain

491 (amino acids 1-147) by EcoR1/Xba1. GFI1B fusion proteins with BirA* were made by

492 subcloning GFI1B-WT, -P2A, - $\triangle$ SNAG (amino acids 21-330), or the SNAG domain (amino

493 acids 1-20) into EcoR1/BamH1 sites in frame to BirA* in the MCS-BiolD2-HA vector, a

494 gift from Kyle Roux (Addgene plasmid \#74224). Subsequently, GFI1B-BirA*:HA forms

495 were expressed from PCR amplified sequences subcloned into pLVX-Tight-Puro

496 (Clontech) with Not1/Mlu1 for doxycycline inducible expression with the Tet-On Advanced

497 (Clontech) system. BirA*:HA only vector was created by inserting a start codon and an

498 EcoR1 site into Age1/BamH1 (5' of BirA*:HA) in pcDNA3.1 and subsequently put into

499 pLVX-Tight-Puro by EcoR1. Primers used for cloning are listed in Table S2. The LSD1

500 construct was a generous gift from Sunil Sharma. Complete open-reading frame

501 sequences for all constructs were confirmed by automated di-deoxy sequencing in the

502 University of Utah DNA sequencing HSC Core Facility. 


\section{Reagents and antibodies}

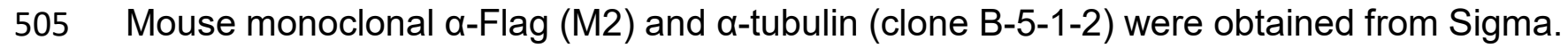

506 Rabbit polyclonal a-HA (ab9110) and a-HMG20B (ab167415) were obtained from Abcam.

507 Rabbit polyclonal a-LSD1 (C69G12) and a-RCOR1 (D6I2U) were obtained from Cell

508 Signaling Technology. Mouse monoclonal $\alpha-H M G 20 A$ (D-5) and $\alpha-G F P$ (B-2) were

509 obtained from Santa Cruz Biotechnology. Rabbit polyclonal a-SMARCB1 (A301-087A)

510 and $\alpha-P H F 21 A$ (A303-603A) were obtained from Bethyl Laboratories and a-SMARCC1

511 (17722-1-AP) was obtained from Proteintech. Mouse monoclonal a-V5 (46-0705) was

512 obtained from Invitrogen. Horse Radish Peroxidase (HRP) conjugated a-mouse (715-

513 035-150) and $\alpha$-rabbit (711-035-152) antibodies were obtained from Jackson

514 Immunoresearch. HRP-conjugated streptavidin (RPN1231) (SAv) was obtained from GE

515 Healthcare UK Limited. APC a-human CD61 (VI-PI.2), and 4',6-diamidino-2-phenylindole,

516 dilactate (DAPI) (422801) were obtained from BioLegend. Restriction endonucleases and

517 ligases were obtained from New England Biosciences. Puromycin (puro), hygromycin

518 (hygro), and G418/Geneticin (Neo) were obtained from Invitrogen. Protein-G Sepharose

5194 Fast Flow and SAv-Sepharose beads were obtained from GE Healthcare Bio-Sciences

$520 \mathrm{AB}$. Biotin was obtained from TCl Chemicals and doxycycline (doxy) was obtained from

521 Fisher Scientific. Benzidine di-hydrochloride, 12-O-Tetradecanoylphorbol-13-acetate

522 (TPA), and polybrene were obtained Sigma. Polyethylenimine (PEI) 25kD Linear Form

523 was obtained from Polysciences (\#23966-2). All other materials were of reagent grade.

525 Transient transfection and co-immunoprecipitation 
526 Transient transfections in COS7L cells were performed using expression plasmids in the

527 indicated combination and Lipofectamine 2000 per the manufacturer's instructions.

528 Monolayers were washed twice with phosphate-buffered saline (PBS), scraped into ice-

529 cold lysis buffer $(50 \mathrm{mM}$ Tris, $150 \mathrm{mM} \mathrm{NaCl}, 10 \%$ glycerol, $1 \mathrm{mM}$ EDTA, $1 \mathrm{mM}$

530 dithiothreitol [DTT], 1\% Triton X-100, 0.1\% SDS, 1 mM phenylmethylsulfonyl fluoride

531 [PMSF], $10 \mu \mathrm{g} / \mathrm{ml}$ aprotinin [pH 8.0]), disrupted by sonication (10 1-second pulses at 5W

532 using Virtis Virsonic sonicator), rocked $30 \mathrm{~min}$ at $4^{\circ} \mathrm{C}$, and clarified by centrifugation. As

533 indicated by the figures, antibodies were added to clarified extracts for 90 min, then the

534 immune complexes were captured by Protein-G-Sepharose beads that were washed in

535 lysis buffer. Immune complexes were washed $4 \mathrm{X}$ with lysis buffer and boiled in SDS-

536 PAGE sample buffer supplemented with DTT.

\section{Immunoblotting}

539 After separation by SDS-PAGE, cleared lysates or immune complexes were examined

540 by immunoblot following standard methods. Briefly, separated protein products were

541 transferred to nitrocellulose filters $(0.45 \mu \mathrm{m}$, Genesee Scientific) using $25 \mathrm{mM}$ Tris, 192

$542 \mathrm{mM}$ glycine $(\mathrm{pH} 8.35)$ for 1 hour at $4^{\circ} \mathrm{C}$. Nitrocellulose filters were blocked with PBS-T (1X

543 PBS, $0.05 \%$ Tween-20) supplemented with $0.25 \%$ gelatin and $0.2 \% \mathrm{NaN}_{3}(\mathrm{GBB})$ and

544 incubated with primary antibodies overnight at $4^{\circ} \mathrm{C}$ as indicated in the figures. Filters were

545 then washed successively with PBS-T and incubated with species-appropriate HRP-

546 conjugated antibody for 1 hour at room temperature. After PBS-T washes, proteins were

547 visualized by chemiluminescence detection (ECL; $30 \mathrm{mM}$ Tris [pH 8.5], $375 \mu \mathrm{M}$ Luminol,

$548 \quad 67.5 \mu \mathrm{M}$ p-Coumaric acid, $0.02 \% \mathrm{H}_{2} \mathrm{O}_{2}$ ) using autoradiography film (Genesee Scientific). 


\section{Luciferase reporter assays}

551 Luciferase assays were performed using the Dual-Luciferase Reporter Assay System

552 (Promega) as per manufacturer's instructions. Briefly, 3.25 x $10^{5}$ 293-T-Rex-5xGal-

553 luciferase cells were plated per well in 6-well plates. Transient transfection of the

554 respective GFI1B:Gal4 expression constructs was performed with Lipofectamine 2000 as

555 per manufacturer's instructions. Reporter activity was measured in cell lysates collected

55648 hours post transfection using a Modulus Microplate reader (Turner BioSystems).

557 Firefly luciferase activity was normalized to constitutively expressed, co-transfected

558 Renilla luciferase control. Statistical significance was determined by two-sided unpaired

$559 \quad t$-tests using GraphPad Prism 7.0.

560

\section{Chemical differentiation and phenotypic assays}

$5624 \times 10^{5} \mathrm{~K} 562$ cells were treated with $30 \mathrm{nM}$ TPA. Cells were harvested 4 days post-

563 induction, washed and incubated with a-CD61 antibody diluted 1:100 in Hanks Buffered

564 Saline Solution (HBSS, Gibco) supplemented with 0.5\% BSA (RPI Corp). Fluorescence

565 signal from DAPI-negative cells was detected by flow cytometry (Fortessa, BD

566 Biosciences) and analyzed using FlowJo analysis software (TreeStar). Heme/porphyrin

567 analysis was analyzed by UPLC with an Acquity UPLC BEH C18, $1.7 \mu \mathrm{M}, 2.1 \times 100 \mathrm{~mm}$

568 column performed at the Iron and Heme Core facility of the University of Utah. Benzidine

569 staining was performed by collecting $1 \times 10^{6} \mathrm{~K} 562$ cells, washing with PBS, and staining

570 with $0.2 \%$ benzidine dihydrochloride in $0.5 \%$ acetic acid for 15 minutes at room

571 temperature. Benzidine positive cells were quantified by hemocytometer using bright-field 
572 microscopy. Experiments were performed in triplicate with 200 cells or more counted from

573 randomly selected fields for each condition. Statistical significance was determined by

574 two-sided unpaired $t$-tests using GraphPad Prism 7.0.

575

576 Streptavidin affinity capture of biotin-modified proteins

577 To induce GFI1B-BirA*:HA in $\mathrm{K}^{*} 62$ cells, $6 \times 10^{7}$ cells were treated with $1 \mu \mathrm{g} / \mathrm{ml}$

578 doxycycline for 48 hours. $30 \mu \mathrm{M}$ biotin was added 16 hours before harvesting. Cells were

579 collected, washed $2 X$ with ice cold PBS, lysed with ice cold lysis buffer (50mM Tris pH7.5,

$580500 \mathrm{mM} \mathrm{NaCl}, 0.5 \mathrm{mM}$ EDTA, 1\% Triton X-100), sonicated and cleared by centrifugation.

581 Biotinylated complexes were captured by SAv-Sepharose High Performance Beads and

582 rocked at $4^{\circ} \mathrm{C}$ overnight. Beads were washed $5 \mathrm{X}$ with lysis buffer supplemented with SDS,

583 boiled in DTT-containing sample buffer and fractionated by SDS-PAGE (10\% T/2.67\% C,

584 4\% Stack). The gel was stained with Colloidal Coomassie Blue (Invitrogen). Lanes were

585 divided into two equal sections for downstream LC-MS-MS analysis.

\section{Protein identification by mass spectrometry}

588 Mass spectrometry was performed by the Taplin Mass Spectrometry Facility, Cell Biology

589 Department, Harvard Medical School. Briefly, samples were reduced using 1 mM DTT in

$59050 \mathrm{mM}$ ammonium bicarbonate for 30 minutes at $60^{\circ} \mathrm{C}$, cooled and $5 \mathrm{mM}$ iodoacetamide

591 in $50 \mathrm{mM}$ ammonium bicarbonate added for 15 minutes in the dark at ambient. 5 mM DTT

592 was added to quench iodoacetamide derivatization. $5 \mathrm{ng} / \mu \mathrm{l}$ of sequencing-grad trypsin

593 (Promega) was added and proteins digested overnight at $37^{\circ} \mathrm{C}$. Samples were then

594 desalted and reconstituted in $5-10 \mu \mathrm{l}$ of HPLC solvent A $(2.5 \%$ acetonitrile, $0.1 \%$ formic 
595 acid). A nano-scale reverse-phase HPLC capillary column was created by packing 2.6

$596 \mu \mathrm{m}$ C18 spherical silica beads into a fused silica capillary $(100 \mu \mathrm{m}$ inner diameter $\mathrm{x} \sim 30$

$597 \mathrm{~cm}$ length) with a flame-drawn tip. After column equilibration, each sample was loaded

598 via a Famos auto sampler (LC Packings) onto the column. A linear gradient was formed

599 to elute peptides with increasing concentrations of solvent B $(97.5 \%$ acetonitrile, $0.1 \%$

600 formic acid). Eluted peptides were subjected to electrospray ionization coupled to an LTQ

601 Orbitrap Velos Pro ion-trap mass spectrometer (Thermo-Fisher Scientific) to produce

602 tandem mass spectra whose $b$ - and $y$-ion series patterns were compared to theoretical

603 digests of the proteome in Sequest (Thermo-Fisher Scientific) to establish protein identity.

604 Data were filtered to a 1-2\% peptide false discovery rate.

605

\section{Statistical analysis of mass spectrometry data}

607 The read intensities were averaged across the biological triplicates for each sample. To 608 establish the GFI1B proximitome (assessment of GFI1B-BirA* vs. BirA* only), sum read

609 intensities among detected proteins were ranked from 0 to 100 percent. A total of 2751

610 proteins were detected in the BirA* only sample and were utilized to established a

611 background intensity ranking. The intensity fold change for each ranked protein was

612 determined by dividing the GFI1B-BirA*:HA average read intensity by the BirA*:HA only

613 average read intensity. This calculated fold change of the intensity was compared to the

614 average intensity rank and was observed to follow a strong diminution of variance with

615 increasing values as expected. To identify proteins with aberrantly increased detection,

$616 z$ z-scores were calculated for each of the 2751 proteins as the deviance in rank from the

617 localized (across the background intensity axis) mean rank compared to the localized 
618 standard deviation of the ranks after the manner of Eubank (96). The corresponding $p$ -

619 value from a $t$-distribution of a 20-protein smoothing selection was determined and utilized

620 to rank prioritize proteins showcasing the most consistent and increased abundance

621 compared to expectation. Proteins with a $p$-value of $<0.05$ were considered hits. This

622 approach was deemed appropriate as background intensity of pertinent proteins varied

623 and detecting relative abundance compared to background was paramount. A volcano

624 plot was created from the - $\log _{10}$ of the $p$-value and the $\log _{2}$ of the intensity fold change.

625 Read intensities detected in GFI1B-BirA*:HA but not in the BirA*:HA only sample (500

626 proteins) were analyzed separately considering only the top 50-100 percentile of ranked

627 GFI1B-BirA*:HA proximity partners as hits (a total of 35 proteins). Likewise, assessment

628 of the GFI1B-BirA*:HA vs. GFI1B- $\triangle$ SNAG-BirA*:HA, GFI1B-BirA*:HA vs. GFI1B-P2A-

629 BirA*:HA, and SNAG-BirA*:HA vs. BirA*:HA only was done by establishing a background

630 percentile ranking of GFI1B- $\triangle$ SNAG-BirA*:HA (2897 proteins), GFI1B-P2A-BirA*:HA

631 (2938 proteins), and BirA*:HA (2751 proteins). Proteins were then plotted according to

632 background rank score and fold change. Statistical analysis was performed as described

633 above for GFI1B-BirA*:HA vs. BirA*:HA using nonparametric regression and spline

634 smoothing (97). SAS version 9.4 and Microsoft Excel were used for statistical calculations

635 and data comparisons.

636

637 ACKNOWLEDGEMENTS

638 The authors thank Katharine Ullman, Jason Gertz, Trudy Oliver, Charles Murtaugh,

639 Rodney Stewart, and Mahesh Chandrasekharan for their helpful insights and suggestions

640 during preparation of this manuscript. We acknowledge the following University of Utah 
641 Core facilities: DNA sequencing and cell line authentication was performed at the DNA

642 Sequencing Core Facility. Oligonucleotides were synthesized by the DNA/Peptide Facility

643 of the Health Sciences Center (HSC). Flow cytometry was performed by the Flow

644 Cytometry Facility with support from the National Cancer Institute (5P30CA042014-24),

645 and heme/porphyrin analysis was performed at the Heme \& Iron Facility supported in part

646 by a grant from the NIH National Institute of Diabetes and Digestive and Kidney Diseases,

647 (U54DK110858). Mass spectrometry was performed at the Taplin Mass Spectrometry

648 Facility, Cell Biology Department, Harvard Medical School with a special thanks to Ross

649 Tomaino.

650 This work was supported by the National Institutes of Health (R01CA201235)

651 (MEE) and T32 DK007115 (DM and MJC), and by grants from the American Cancer

652 Society, Alex's Lemonade Stand Foundation and the Hyundai Hope on Wheels

653 Foundation. Supporters had no role in study design, data collection and interpretation, or

654 the decision to submit the work for publication. The authors declare no conflicts of interest

655 associated with the manuscript.

656

657 REFERENCES

658 1. Feinberg AP, Koldobskiy MA, Göndör A. 2016. Epigenetic modulators, modifiers and 659 mediators in cancer aetiology and progression. Nat Rev Genet 17:284-299.

660 2. Avgustinova A, Benitah SA. 2016. Epigenetic control of adult stem cell function. Nat Rev Mol $661 \quad$ Cell Biol 17:643-658.

662 3. Ntziachristos P, Abdel-Wahab O, Aifantis I. 2016. Emerging concepts of epigenetic 663 dysregulation in hematological malignancies. Nature Immunology 17:1016-1024. 
664 4. Egger G, Liang G, Aparicio A, Jones PA. 2004. Epigenetics in human disease and prospects 665 for epigenetic therapy. Nature 429:457-463.

666 5. van der Meer LT, Jansen JH, van der Reijden BA. 2010. Gfi1 and Gfi1b: key regulators of 667 hematopoiesis. Leukemia 24:1834-1843.

668 6. Lancrin C, Mazan M, Stefanska M, Patel R, Lichtinger M, Costa G, Vargel O, Wilson NK, 669 Möröy T, Bonifer C, Göttgens B, Kouskoff V, Lacaud G. 2012. GFI1 and GFI1B control the 670 loss of endothelial identity of hemogenic endothelium during hematopoietic commitment. $671 \quad$ Blood 120:314-322.

672 7. Zeng H, Yücel R, Kosan C, Klein-Hitpass L, Möröy T. 2004. Transcription factor Gfi1 regulates 673 self-renewal and engraftment of hematopoietic stem cells. EMBO J 23:4116-4125.

674 8. Hock H, Hamblen MJ, Rooke HM, Schindler JW, Saleque S, Fujiwara Y, Orkin SH. 2004. Gfi6751 restricts proliferation and preserves functional integrity of haematopoietic stem cells. Nature 431:1002-1007.

9. Yücel R, Karsunky H, Klein-Hitpass L, Möröy T. 2003. The transcriptional repressor Gfi1 affects development of early, uncommitted c-Kit+ T cell progenitors and CD4/CD8 lineage decision in the thymus. J Exp Med 197:831-844.

10. Fiolka K, Hertzano R, Vassen L, Zeng H, Hermesh O, Avraham KB, Dührsen U, Möröy T. 2006. Gfi1 and Gfi1b act equivalently in haematopoiesis, but have distinct, non-overlapping functions in inner ear development. EMBO reports 7:326-333.

11. Karsunky H, Zeng H, Schmidt T, Zevnik B, Kluge R, Schmid KW, Dührsen U, Möröy T. 2002. Inflammatory reactions and severe neutropenia in mice lacking the transcriptional repressor Gfi1. Nat Genet 30:295-300.

12. Hock H, Hamblen MJ, Rooke HM, Traver D, Bronson RT, Cameron S, Orkin SH. 2003. Intrinsic requirement for zinc finger transcription factor Gfi-1 in neutrophil differentiation. Immunity 18:109-120. 
13. Zörnig M, Schmidt T, Karsunky H, Grzeschiczek A, Moroy T. 1996. Zinc finger protein GFI-1 cooperates with myc and pim-1 in T-cell lymphomagenesis by reducing the requirements for IL-2. Oncogene 12:1789-1801.

14. Khandanpour C, Thiede C, Valk PJM, Sharif-Askari E, Nückel H, Lohmann D, Horsthemke B, 693 Siffert W, Neubauer A, Grzeschik K-H, Bloomfield CD, Marcucci G, Maharry K, Slovak ML, 694 Van der Reijden BA, Jansen JH, Schackert HK, Afshar K, Schnittger S, Peeters JK, 695 Kroschinsky F, Ehninger G, Lowenberg B, Dührsen U, Möröy T. 2010. A variant allele of Growth Factor Independence 1 (GFI1) is associated with acute myeloid leukemia. Blood 115:2462-2472.

15. Khandanpour C. 2017. Growth factor independence 1 (Gfi1) regulates the AML supporting function of mesenchymal stromal cells. Experimental Hematology 53:S90.

16. Möröy T. 2014. The zinc finger protein Gfi1 maintains development and progression of lymphoid leukemia by blocking the activation of the tumor suppressor p53. Experimental Hematology 42:S7.

17. Volpe G, Walton DS, Grainger DE, Ward C, Cauchy P, Blakemore D, Coleman DJL, Cockerill PN, Garcia P, Frampton J. 2017. Prognostic significance of high GFI1 expression in AML of normal karyotype and its association with a FLT3-ITD signature. Sci Rep 7:11148.

18. Xia J, Bolyard AA, Rodger E, Stein S, Aprikyan AA, Dale DC, Link DC. 2009. Prevalence of mutations in ELANE, GFI1, HAX1, SBDS, WAS and G6PC3 in patients with severe congenital neutropenia. Br J Haematol 147:535-542.

19. Person RE, Li F-Q, Duan Z, Benson KF, Wechsler J, Papadaki HA, Eliopoulos G, Kaufman C, Bertolone SJ, Nakamoto B, Papayannopoulou T, Grimes HL, Horwitz M. 2003. Mutations in proto-oncogene GFI1 cause human neutropenia and target ELA2. Nat Genet 34:308-312. controls human erythroid and megakaryocytic differentiation by regulating TGF- signaling at the bipotent erythro-megakaryocytic progenitor stage. Blood 115:2784-2795. 
715

716

717

718

719

720

721

722

723

724

725

726

727

728

729

730

731

732

733

734

735

736

737

738

739

740

21. Vassen L, Beauchemin H, Lemsaddek W, Krongold J, Trudel M, Möröy T. 2014. Growth factor independence $1 \mathrm{~b}$ (gfi1b) is important for the maturation of erythroid cells and the regulation of embryonic globin expression. PLoS ONE 9:e96636.

22. Singh D, Upadhyay G, Sengupta A, Biplob MA, Chakyayil S, George T, Saleque S. 2016. Cooperative Stimulation of Megakaryocytic Differentiation by Gfi1b Gene Targets Kindlin3 and Talin1. PLoS ONE 11:e0164506.

23. Beauchemin H, Shooshtarizadeh P, Vadnais C, Vassen L, Pastore YD, Möröy T. 2017. Gfi1b controls integrin signaling-dependent cytoskeleton dynamics and organization in megakaryocytes. Haematologica 102:484-497.

24. Saleque S, Cameron S, Orkin SH. 2002. The zinc-finger proto-oncogene Gfi-1b is essential for development of the erythroid and megakaryocytic lineages. Genes Dev 16:301-306.

25. Stevenson WS, Morel-Kopp M-C, Chen Q, Liang HP, Bromhead CJ, Wright S, Turakulov R, Ng AP, Roberts AW, Bahlo M, Ward CM. 2013. GFI1B mutation causes a bleeding disorder with abnormal platelet function. J Thromb Haemost 11:2039-2047.

26. Monteferrario D, Bolar NA, Marneth AE, Hebeda KM, Bergevoet SM, Veenstra H, Laros-van Gorkom BAP, MacKenzie MA, Khandanpour C, Botezatu L, Fransen E, Van Camp G, Duijnhouwer AL, Salemink S, Willemsen B, Huls G, Preijers F, Van Heerde W, Jansen JH, Kempers MJE, Loeys BL, Van Laer L, Van der Reijden BA. 2014. A dominant-negative GFI1B mutation in the gray platelet syndrome. N Engl J Med 370:245-253.

27. Uchiyama Y, Ogawa Y, Kunishima S, Shiina M, Nakashima M, Yanagisawa K, Yokohama A, Imagawa E, Miyatake S, Mizuguchi T, Takata A, Miyake N, Ogata K, Handa H, Matsumoto N. 2018. A novel GFI1B mutation at the first zinc finger domain causes congenital macrothrombocytopenia. Br J Haematol 181:843-847.

28. Kitamura K, Okuno Y, Yoshida K, Sanada M, Shiraishi Y, Muramatsu H, Kobayashi R, Furukawa K, Miyano S, Kojima S, Ogawa S, Kunishima S. 2016. Functional characterization of a novel GFI1B mutation causing congenital macrothrombocytopenia. J Thromb Haemost. 
741 29. Vassen L, Khandanpour C, Ebeling P, Van der Reijden BA, Jansen JH, Mahlmann S, Dührsen

742 U, Möröy T. 2009. Growth factor independent 1b (Gfi1b) and a new splice variant of Gfi1b are

743 highly expressed in patients with acute and chronic leukemia. Int J Hematol 89:422-430.

744 30. Elmaagacli AH, Koldehoff M, Zakrzewski JL, Steckel NK, Ottinger H, Beelen DW. 2007.

745 Growth factor-independent 1B gene (GFI1B) is overexpressed in erythropoietic and 746 megakaryocytic malignancies and increases their proliferation rate. $\mathrm{Br} \mathrm{J}$ Haematol 136:212219.

748 31. Hernández A, Villegas A, Anguita E. 2010. Human promoter mutations unveil Oct-1 and 749 GATA-1 opposite action on Gfi1b regulation. Annals of Hematology 89:759-765.

750 32. Tong B, Grimes HL, Yang TY, Bear SE, Qin Z, Du K, El-Deiry WS, Tsichlis PN. 1998. The 751 Gfi-1B proto-oncoprotein represses p21WAF1 and inhibits myeloid cell differentiation. Mol $752 \quad$ Cell Biol 18:2462-2473.

753 33. Thivakaran A, Botezatu L, Hönes JM, Schütte J, Vassen L, Al-Matary YS, Patnana P, Zeller

754 A, Heuser M, Thol F, Gabdoulline R, Olberding N, Frank D, Suslo M, Köster R, Lennartz K, 755 Görgens A, Giebel B, Opalka B, Dührsen U, Khandanpour C. 2018. Gfi1b: a key player in the 756 genesis and maintenance of acute myeloid leukemia and myelodysplastic syndrome. $757 \quad$ Haematologica 103:614-625.

758 34. Möröy T, Vassen L, Wilkes B, Khandanpour C. 2015. From cytopenia to leukemia: the role of 759 Gfi1 and Gfi1b in blood formation. Blood 126:2561-2569.

760 35. Zweidler-McKay PA, Grimes HL, Flubacher MM, Tsichlis PN. 1996. Gfi-1 encodes a nuclear 761 zinc finger protein that binds DNA and functions as a transcriptional repressor. Mol Cell Biol $762 \quad 16: 4024-4034$.

763 36. Lee S, Doddapaneni K, Hogue A, McGhee L, Meyers S, Wu Z. 2010. Solution structure of Gfi7641 zinc domain bound to consensus DNA. J Mol Biol 397:1055-1066.

765 37. Anguita E, Villegas A, Iborra F, Hernández A. 2010. GFI1B controls its own expression binding 766 to multiple sites. Haematologica 95:36-46. 
767

768

769

770

771

772

773

774

775

776

777

778

779

780

781

782

783

784

785

786

787

788

789

790

791

792

38. Nakazawa Y, Suzuki M, Manabe N, Yamada T, Kihara-Negishi F, Sakurai T, Tenen DG, Iwama A, Mochizuki M, Oikawa T. 2007. Cooperative interaction between ETS1 and GFI1 transcription factors in the repression of Bax gene expression. Oncogene 26:3541-3550.

39. Huang D-Y, Kuo Y-Y, Chang Z-F. 2005. GATA-1 mediates auto-regulation of Gfi-1B transcription in K562 cells. Nucleic Acids Research 33:5331-5342.

40. Marteijn JAF, van der Meer LT, van Emst L, van Reijmersdal S, Wissink W, de Witte T, Jansen JH, Van der Reijden BA. 2007. Gfi1 ubiquitination and proteasomal degradation is inhibited by the ubiquitin ligase Triad1. Blood 110:3128-3135.

41. Dahl R, lyer SR, Owens KS, Cuylear DD, Simon MC. 2007. The transcriptional repressor GFI1 antagonizes PU.1 activity through protein-protein interaction. J Biol Chem 282:6473-6483.

42. Andrade D, Velinder M, Singer J, Maese L, Bareyan D, Nguyen H, Chandrasekharan MB, Lucente H, McClellan D, Jones D, Sharma S, Liu F, Engel ME. 2016. SUMOylation Regulates Growth Factor Independence 1 in Transcriptional Control and Hematopoiesis. Mol Cell Biol 36:1438-1450.

43. Saleque S, Kim J, Rooke HM, Orkin SH. 2007. Epigenetic regulation of hematopoietic differentiation by Gfi-1 and Gfi-1b is mediated by the cofactors CoREST and LSD1. Mol Cell 27:562-572.

44. Lin Y, Wu Y, Li J, Dong C, Ye X, Chi Y-I, Evers BM, Zhou BP. 2010. The SNAG domain of Snail 1 functions as a molecular hook for recruiting lysine-specific demethylase 1. EMBO J 29:1803-1816.

45. Laurent B, Randrianarison-Huetz V, Frisan E, Andrieu-Soler C, Soler E, Fontenay M, Dusanter-Fourt I, Duménil D. 2012. A short Gfi-1B isoform controls erythroid differentiation by recruiting the LSD1-CoREST complex through the dimethylation of its SNAG domain. J Cell Sci 125:993-1002.

46. Velinder M, Singer J, Bareyan D, Meznarich J, Tracy CM, Fulcher JM, McClellan D, Lucente H, Franklin S, Sharma S, Engel ME. 2017. GFI1 functions in transcriptional control and cell 
fate determination require SNAG domain methylation to recruit LSD1. Biochem J 474:29512951.

47. Upadhyay G, Chowdhury AH, Vaidyanathan B, Kim D, Saleque S. 2014. Antagonistic actions of Rcor proteins regulate LSD1 activity and cellular differentiation. Proc Natl Acad Sci USA 111:8071-8076.

48. Shi Y, Lan F, Matson C, Mulligan P, Whetstine JR, Cole PA, Casero RA, Shi Y. 2004. Histone demethylation mediated by the nuclear amine oxidase homolog LSD1. Cell 119:941-953.

49. Metzger E, Wissmann M, Yin N, Müller JM, Schneider R, Peters AHFM, Günther T, Buettner R, Schüle R. 2005. LSD1 demethylates repressive histone marks to promote androgenreceptor-dependent transcription. Nature 437:436-439.

50. Huang J, Sengupta R, Espejo AB, Lee MG, Dorsey JA, Richter M, Opravil S, Shiekhattar R, Bedford MT, Jenuwein T, Berger SL. 2007. p53 is regulated by the lysine demethylase LSD1. Nature 449:105-108.

51. Yang J, Huang J, Dasgupta M, Sears N, Miyagi M, Wang B, Chance MR, Chen X, Du Y, Wang Y, An L, Wang Q, Lu T, Zhang X, Wang Z, Stark GR. 2010. Reversible methylation of

52. Zhang X, Tanaka K, Yan J, Li J, Peng D, Jiang Y, Yang Z, Barton MC, Wen H, Shi X. 2013. promoter-bound STAT3 by histone-modifying enzymes. Proc Natl Acad Sci USA 107:21499_ 21504. Regulation of estrogen receptor $\alpha$ by histone methyltransferase SMYD2-mediated protein methylation. Proc Natl Acad Sci USA 110:17284-17289.

53. Cho H-S, Suzuki T, Dohmae N, Hayami S, Unoki M, Yoshimatsu M, Toyokawa G, Takawa M, Chen T, Kurash JK, Field HI, Ponder BAJ, Nakamura Y, Hamamoto R. 2011. Demethylation of RB regulator MYPT1 by histone demethylase LSD1 promotes cell cycle progression in cancer cells. Cancer Res 71:655-660. 
817

818

819

820

821

822

823

824

825

826

827

828

829

830

831

832

833

834

835

836

837

838

839

840

841

842

54. Lee J-Y, Park J-H, Choi H-J, Won H-Y, Joo H-S, Shin D-H, Park MK, Han B, Kim KP, Lee TJ, Croce CM, Kong G. 2017. LSD1 demethylates HIF1a to inhibit hydroxylation and ubiquitinmediated degradation in tumor angiogenesis. Oncogene 36:5512-5521.

55. Wang J, Hevi S, Kurash JK, Lei H, Gay F, Bajko J, Su H, Sun W, Chang H, Xu G, Gaudet F, Li E, Chen T. 2009. The lysine demethylase LSD1 (KDM1) is required for maintenance of global DNA methylation. Nat Genet 41:125-129.

56. Kontaki H, Talianidis I. 2010. Lysine methylation regulates E2F1-induced cell death. Mol Cell 39:152-160.

57. Zhang C, Hoang N, Leng F, Saxena L, Lee L, Alejo S, Qi D, Khal A, Sun H, Lu F, Zhang H. 2018. LSD1 demethylase and the methyl-binding protein PHF20L1 prevent SET7 methyltransferase-dependent proteolysis of the stem-cell protein SOX2. J Biol Chem 293:3663-3674.

58. Wang Y, Zhang H, Chen Y, Sun Y, Yang F, Yu W, Liang J, Sun L, Yang X, Shi L, Li R, Li Y, Zhang Y, Li Q, Yi X, Shang Y. 2009. LSD1 is a subunit of the NuRD complex and targets the metastasis programs in breast cancer. Cell 138:660-672.

59. Yang Y, Huang W, Qiu R, Liu R, Zeng Y, Gao J, Zheng Y, Hou Y, Wang S, Yu W, Leng S, Feng D, Wang Y. 2018. LSD1 coordinates with the SIN3A/HDAC complex and maintains sensitivity to chemotherapy in breast cancer. J Mol Cell Biol 387:49.

60. Ray SK, Li HJ, Metzger E, Schüle R, Leiter AB. 2014. CtBP and associated LSD1 are required for transcriptional activation by NeuroD1 in gastrointestinal endocrine cells. Mol Cell Biol $34: 2308-2317$.

61. Tsai M-C, Manor O, Wan Y, Mosammaparast N, Wang JK, Lan F, Shi Y, Segal E, Chang HY. 2010. Long noncoding RNA as modular scaffold of histone modification complexes. Science 329:689-693.

62. Shi Y-J, Matson C, Lan F, Iwase S, Baba T, Shi Y. 2005. Regulation of LSD1 histone demethylase activity by its associated factors. Mol Cell 19:857-864. 
843 63. Roux KJ, Kim DI, Raida M, Burke B. 2012. A promiscuous biotin ligase fusion protein identifies

844 proximal and interacting proteins in mammalian cells. J Cell Biol 196:801-810.

845 64. Kim DI, Jensen SC, Noble KA, Kc B, Roux KH, Motamedchaboki K, Roux KJ. 2016. An

846 improved smaller biotin ligase for BiolD proximity labeling. Mol Biol Cell 27:1188-1196.

847 65. Hakimi M-A, Bochar DA, Chenoweth J, Lane WS, Mandel G, Shiekhattar R. 2002. A core-

848 BRAF35 complex containing histone deacetylase mediates repression of neuronal-specific

849 genes. Proc Natl Acad Sci USA 99:7420-7425.

850 66. Gocke CB, Yu H. 2008. ZNF198 stabilizes the LSD1-CoREST-HDAC1 complex on chromatin

$851 \quad$ through its MYM-type zinc fingers. PLoS ONE 3:e3255.

852 67. Rivero S, Ceballos-Chávez M, Bhattacharya SS, Reyes JC. 2015. HMG20A is required for 853 SNAI1-mediated epithelial to mesenchymal transition. Oncogene 34:5264-5276.

854 68. Garçon L, Lacout C, Svinartchouk F, Le Couédic J-P, Villeval J-L, Vainchenker W, Duménil

855 D. 2005 . Gfi-1B plays a critical role in terminal differentiation of normal and transformed 856 erythroid progenitor cells. Blood 105:1448-1455.

857 69. Lam LT, Ronchini C, Norton J, Capobianco AJ, Bresnick EH. 2000. Suppression of erythroid 858 but not megakaryocytic differentiation of human K562 erythroleukemic cells by notch-1. J Biol 859 Chem 275:19676-19684.

860 70. Meng Y-S, Khoury H, Dick JE, Minden MD. 2005. Oncogenic potential of the transcription 861 factor LYL1 in acute myeloblastic leukemia. Leukemia 19:1941-1947.

862 71. Maiques-Diaz A, Somervaille TC. 2016. LSD1: biologic roles and therapeutic targeting. $863 \quad$ Epigenomics 8:1103-1116.

864 72. Kim DI, Roux KJ. 2016. Filling the Void: Proximity-Based Labeling of Proteins in Living Cells. 865 Trends Cell Biol 26:804-817.

866 73. Leon-Del-Rio A, Gravel RA. 1994. Sequence requirements for the biotinylation of carboxyl867 terminal fragments of human propionyl-CoA carboxylase alpha subunit expressed in $868 \quad$ Escherichia coli. J Biol Chem 269:22964-22968. 
74. Vassen L. 2005. Direct transcriptional repression of the genes encoding the zinc-finger proteins Gfi1b and Gfi1 by Gfi1b. Nucleic Acids Research 33:987-998.

871 75. Rodriguez P, Bonte E, Krijgsveld J, Kolodziej KE, Guyot B, Heck AJR, Vyas P, de Boer E,

872 Grosveld F, Strouboulis J. 2005. GATA-1 forms distinct activating and repressive complexes 873 in erythroid cells. EMBO J 24:2354-2366.

874 76. Kuo Y-Y, Chang Z-F. 2007. GATA-1 and Gfi-1B interplay to regulate Bcl-xL transcription. Mol $875 \quad$ Cell Biol 27:4261-4272.

876 77. Jegalian AG, Wu H. 2002. Regulation of Socs gene expression by the proto-oncoprotein GFI877 1B: two routes for STAT5 target gene induction by erythropoietin. J Biol Chem 277:23458782352.

879 78. Chowdhury AH, Ramroop JR, Upadhyay G, Sengupta A, Andrzejczyk A, Saleque S. 2013.

880 Differential transcriptional regulation of meis1 by Gfi1b and its co-factors LSD1 and CoREST.

$881 \quad$ PLoS ONE 8:e53666.

882 79. Vassen L, Fiolka K, Möröy T. 2006. Gfi1b alters histone methylation at target gene promoters and sites of gamma-satellite containing heterochromatin. EMBO J 25:2409-2419.

884 80. Ceballos-Chávez M, Rivero S, García-Gutiérrez P, Rodríguez-Paredes M, García-Domínguez M, Bhattacharya S, Reyes JC. 2012. Control of neuronal differentiation by sumoylation of BRAF35, a subunit of the LSD1-CoREST histone demethylase complex. Proc Natl Acad Sci USA 109:8085-8090.

81. Esteghamat F, van Dijk TB, Braun H, Dekker S, van der Linden R, Hou J, Fanis P, Demmers J, van IJcken W, Ozgür Z, Horos R, Pourfarzad F, Lindern von M, Philipsen S. 2011. The DNA binding factor Hmg20b is a repressor of erythroid differentiation. Haematologica 96:1252-

82. Hock R, Furusawa T, Ueda T, Bustin M. 2007. HMG chromosomal proteins in development and disease. Trends Cell Biol 17:72-79. 
894 83. Malarkey CS, Churchill MEA. 2012. The high mobility group box: the ultimate utility player of 895 a cell. Trends Biochem Sci 37:553-562.

896 84. Wynder C, Hakimi M-A, Epstein JA, Shilatifard A, Shiekhattar R. 2005. Recruitment of MLL 897 by HMG-domain protein iBRAF promotes neural differentiation. Nat Cell Biol 7:1113-1117.

898 85. Chai P, Tian J, Zhao D, Zhang H, Cui J, Ding K, Liu B. 2016. GSE1 negative regulation by 899 miR-489-5p promotes breast cancer cell proliferation and invasion. Biochem Biophys Res $900 \quad$ Commun 471:123-128.

901 86. Ding K, Tan S, Huang X, Wang X, Li X, Fan R, Zhu Y, Lobie PE, Wang W, Wu Z. 2018. GSE1

902 predicts poor survival outcome in gastric cancer patients by SLC7A5 enhancement of tumor 903 growth and metastasis. J Biol Chem 293:3949-3964.

904 87. Lambert SA, Jolma A, Campitelli LF, Das PK, Yin Y, Albu M, Chen X, Taipale J, Hughes TR, 905 Weirauch MT. 2018. The Human Transcription Factors. Cell 172:650-665.

906 88. Clapier CR, Cairns BR. 2009. The biology of chromatin remodeling complexes. Annu Rev $907 \quad$ Biochem 78:273-304.

908 89. Grimes HL, Chan TO, Zweidler-McKay PA, Tong B, Tsichlis PN. 1996. The Gfi-1 proto909 oncoprotein contains a novel transcriptional repressor domain, SNAG, and inhibits G1 arrest 910 induced by interleukin-2 withdrawal. Mol Cell Biol 16:6263-6272.

911 90. Chen Y, Yang Y, Wang F, Wan K, Yamane K, Zhang Y, Lei M. 2006. Crystal structure of 912 human histone lysine-specific demethylase 1 (LSD1). Proc Natl Acad Sci USA 103:13956$913 \quad 13961$.

914 91. Yang M, Gocke CB, Luo X, Borek D, Tomchick DR, Machius M, Otwinowski Z, Yu H. 2006. 915 Structural basis for CoREST-dependent demethylation of nucleosomes by the human LSD1 916 histone demethylase. Mol Cell 23:377-387.

917 92. Ouyang J, Shi Y, Valin A, Xuan Y, Gill G. 2009. Direct binding of CoREST1 to SUMO-2/3 918 contributes to gene-specific repression by the LSD1/CoREST1/HDAC complex. Mol Cell $919 \quad 34: 145-154$. 
920 93. Nalawansha DA, Pflum MKH. 2017. LSD1 Substrate Binding and Gene Expression Are

921 Affected by HDAC1-Mediated Deacetylation. ACS Chem Biol 12:254-264.

922 94. Holmes A, Roseaulin L, Schurra C, Waxin H, Lambert S, Zaratiegui M, Martienssen RA,

923 Arcangioli B. 2012. Lsd1 and Isd2 control programmed replication fork pauses and imprinting 924 in fission yeast. Cell Rep 2:1513-1520.

925 95. Sumoy L, Carim L, Escarceller M, Nadal M, Gratacòs M, Pujana MA, Estivill X, Peral B. 2000.

926 HMG20A and HMG20B map to human chromosomes $15 q 24$ and 19p13.3 and constitute a 927 distinct class of HMG-box genes with ubiquitous expression. Cytogenet Cell Genet 88:62-67.

928 96. Eubank RL. 1999. Nonparametric Regression and Spline Smoothing, Second Edition. CRC $929 \quad$ Press.

930 97. Ke C, Wang Y. 2004. Smoothing Spline Nonlinear Nonparametric Regression Models. Journal 931 of the American Statistical Association 99:1166-1175.

933 FIGURE AND TABLE LEGENDS

934 Figure 1. LSD1 depletion impedes GFI1B-mediated erythroid differentiation. (A)

935 GFI1B overexpression promotes K562 cell hemoglobinization. Cells were infected with 936 empty vector (EV) or virus expressing GFI1B. GFI1B-expressing cells become red and 937 are benzidine positive. Benzidine positive cells are quantified as mean \pm SD from four 938 biological replicates. (B) TPA triggers cell surface expression of CD61 in K562 cells. 939 CD61 expression was quantified in vehicle- vs. TPA-treated viable cells by flow cytometry. 940 (C) LSD1 depletion and GFI1B overexpression in K562 cells. Small hairpin RNAs 941 targeting LSD1 (LSD1 shRNA) or content-matched scramble control (Scram shRNA) 942 were inducibly transcribed in K562 cells followed by infection at day +3 with GFI1B:flag943 expressing virus or EV. LSD1 and GFI1B were quantified by western blot of whole cell 944 Iysates (WCL). Tubulin served as a loading control. (D) LSD1 depletion blocks GFI1B- 
945 mediated K562 cell hemoglobinization. (E) LSD1 depletion enables TPA-induced CD61

946 expression in the context of GFI1B overexpression. (F-G) LSD1 depletion impairs

947 expression of erythroid fate genes in response to GFI1B overexpression. Alpha- and

948 Beta-globins $(H B A 1 / 2$ and $H B B)$, alpha globin stabilizing protein $(A H S P)$, aminolevulinic

949 acid synthase $(A L A S) 2$, ferrochelatase $(F E C H)$ and glycophorin $(G Y P) A$ expression are

950 shown. (H) LSD1 depletion restores expression of GFI1B-repressed target genes.

951 CDKN1A, GFI1B and MYB expression are shown. Gene expression is quantified by RT-

952 qPCR normalized to GUS. Results are expressed as mean \pm 2 SD from two independent

953 experiments performed in triplicate. ${ }^{*} p<0.05 ;{ }^{* *} p<0.005 ;{ }^{* *} p<0.0005,{ }^{* * * *} p<0.00005$.

955 Figure 2. LSD1 binding is required for GFI1B-regulated cell fate decisions in K562

956 cells. (A) P2A substitution in GFI1B impairs GFI1B_LSD1 binding. K562 cells were

957 transduced with constructs expressing wild type GFI1B, its P2A variant or empty vector

958 (EV) as shown. GFI1B forms were immune purified with $\alpha$-flag (M2) antibody and Protein-

959 G Sepharose. LSD1 was quantified in immune complexes (IC) by western blot. Equal

960 expression (LSD1 and GFI1B) and precipitation (GFI1B) were evaluated by western blot

961 of whole cell lysates (WCL) and ICs, respectively. Tubulin was used to confirm equal gel

962 loading. (B) P2A substitution abolishes transcriptional repression by GFI1B. 293-T-Rex-

$9635 \times$ Gal-luciferase cells were transfected with WT or P2A variants of GFI1B- $\Delta 1 \mathrm{C}: \mathrm{Gal} 4$ (left)

964 or SNAG:Gal4 (right) fusion proteins. Firefly luciferase was measured and normalized to

965 constitutively expressed, co-transfected Renilla luciferase. Reporter activity is expressed

966 as mean \pm SD from two experiments performed in triplicate. (C) P2A substitution impairs

967 GFI1B-mediated hemoglobinization. Benzidine staining was quantified as in $1 \mathrm{~A}$. 
968 Protoporphyrin IX (PPIX) and heme were quantified by UPLC in K562 cells transduced with EV, GFI1B-WT or GFI1B-P2A. (D-F) P2A substitution impairs expression of erythroid

970 fate genes in K562 cells. Expression of $\beta$-globin cluster genes, AHSP, ALAS2, FECH,

971 GYPA and Kruppel-like factor $(K L F) 1$ are shown. (G) P2A substitution abolishes GFI1B-

972 mediated suppression of TPA-induced CD61 expression. Cell surface expression of

973 CD61 in TPA treated cells expressing wild type GFI1B (WT) or its P2A variant was

974 determined by flow cytometry. (H) P2A substitution reverses repression of GFI1B target

975 genes GFI1B, CDKN1A and MYB. Results expressed as mean \pm 2 SD. *,$p<0.05 ;{ }^{* *}, p<$

$9760.005 ;{ }^{* * *}, p<0.0005 ;{ }^{* * *}, p<0.00005$.

977

978 Figure 3. GFIB-BirA* defines a GFI1B proximitome. (A) Schematic representation of

979 strategy for biotin modification of GFI1B partners. GFI1B is fused to the promiscuous 980 biotin ligase with an HA epitope tag, BirA*:HA to form GFI1B-BirA*:HA. GFI1B partners 981 are spontaneously biotinylated due to their proximity to BirA*:HA anchored to GFI1B in 982 cis. Biotinylated products are captured on streptavidin (SAv)-conjugated beads for 983 downstream analysis via candidate-based or screening approaches. (B) BirA*:HA and 984 GFI1B-BirA*:HA fusion biotinylate diverse targets in K562 cells. Cells were transduced 985 with constructs inducibly expressing GFI1B-BirA*:HA, BirA*:HA or EV, treated with 986 doxycycline and harvested. GFI1B-BirA*:HA and BirA*:HA expression was confirmed by $987 \alpha-\mathrm{HA}$ western blot and biotinylated proteins detected by SAv:HRP. Tubulin served as a 988 loading control. (C) GFI1B interacting protein, LSD1 and RCOR1, are enriched among 989 proteins biotinylated by GFI1B-BirA*:HA compared to BirA* or EV., Biotin-modified 990 proteins were collected on SAv-Sepharose. LSD1, RCOR1 and the BirA* fusion proteins 
991 themselves were quantified among purified proteins (SAv P) by western blot. Equal

992 expression was confirmed by western blot in whole cell lysates (input). (D) GFI1B-

993 BirA*:HA identifies members of a GFI1B proximitome. Factors enriched among 994 biotinylated and SAv-Sepharose purified proteins modified by GFI1-BirA*:HA (test) 995 compared to BirA*:HA (control). Fold enrichment is plotted logarithmically as a ratio of 996 average sum read intensities between test and control samples against their $p$-values 997 calculated from their 20 closest ranked neighbors in the data set as described in Materials 998 and Methods. Individual proteins are labeled with their respective gene names. Inset 999 shows an expanded view of the data contained in the boxed area.

1001 Figure 4. Components of transcriptional repression and chromatin remodeling complexes identified by GFI1B proximity labeling. A roster of transcriptionally relevant

1003 complexes and their component members are shown. Proteins are listed according to 1004 HUGO gene nomenclature. With respect to the GFI1B proximitome (GFI1B-BirA*:HA vs. 1005 BirA*:HA), proteins present and enriched-white letters on black background. Those 1006 present by not significantly enriched $(p<0.05)$ —black letters on gray background. Not 1007 present (average sum read intensity below that for EV)_black letters on white 1008 background. Where appropriate, complexes are subdivided into core and putative 1009 members, and/or with canonical vs. non-canonical designations.

1011 Figure 5. LSD1 non-binding variants of GFI1B-BirA* fail to biotinylate LSD1 and

1012 RCOR1. (A) Graphical representation of GFI1B-BirA*:HA fusion proteins employed in 1013 experiments. Each fusion protein is comprised of the GFI1B regions shown in frame with 
1014 the BirA* expression cassette and a C-terminal HA epitope tag to insure equivalent

1015 expression of all forms. The BirA*:HA construct lacks GFI1B structures and instead

1016 begins with an ATG. Wild type (WT) GFI1B is shown. The SNAG and zinc finger (ZnF)

1017 domains (1-6) of GFI1B are shown as black boxes. A thin line represents the GFI1B linker

1018 and connections between ZnFs. The proline to alanine substitution (P2A) in the GFI1B

1019 SNAG domain is indicated with an *. $\triangle$ SNAG represents deletion of the SNAG domain

1020 but preservation of the remaining GFI1B primary structure. (B) Expression of GFI1B-

1021 BirA*:HA forms and proteome-wide biotin modification in K562 cells. K562 cells were

1022 transduced with lentivirus to inducibly express GFI1B-BirA*:HA forms shown and stable

1023 isolates selected as polyclonal populations. Doxycycline-inducible expression of each

1024 fusion protein was confirmed by western blot against the common HA epitope tag and

1025 biotinylating activity confirmed proteome-wide by SAv:HRP detection of transblotted total

1026 cellular protein. Tubulin served as a loading control. (C) LSD1 and RCOR1 are enriched

1027 among biotinylated proteins generated by BirA*:HA fusions competent for LSD1 binding.

1028 Biotin-modified proteins from K562 cells transduced with BirA*:HA fusion proteins shown

1029 were purified from whole cell extracts (SAv P), fractionated by SDS-PAGE and subjected

1030 to western blot with $\alpha$-LSD1, $\alpha$-RCOR1 and $\alpha-H A$ antibodies. Equivalent expression of

1031 each protein in whole cell lysates (Input) was confirmed by western blot with these same 1032 antibodies.

1033

1034 Figure 6. GFI1B recruits the BHC complex in an LSD1-depend manner via the SNAG

1035 domain. K562 cells stably and inducibly expressing the BirA*:HA fusion proteins shown

1036 in 5A were deployed to define GFI1B proximity partners whose recruitment is LSD1- 
1037 dependent. Fusion protein expression was induced by doxycycline addition and 1038 biotinylation permitted in situ. Biotin-modified proteins were captured on SAv-Sepharose 1039 and analyzed by LC-MS-MS as described in Materials and Methods. (A-C) Dot plots are 1040 shown comparing fold change in average sum read intensity among fusion proteins with 1041 intact SNAG domains and capable of LSD1-binding (GFI1B-BirA*:HA and SNAG1042 BirA*:HA) to those with disrupted or absent SNAG domains and deficient in LSD1 binding 1043 (GFI1B-P2A-BirA*:HA, GFI1B- $\triangle$ SNAG-BirA*:HA, and BirA*:HA). For each comparison 1044 (A-GFI1B-BirA*:HA vs. GFI1B- $\triangle$ SNAG-BirA*:HA, B-GFI1B-BirA* vs. GFI1B-P2A1045 BirA*:HA, and C-SNAG-BirA*:HA vs. BirA*:HA), average sum read intensities were 1046 ranked according to the LSD1-nonbinding sample and plotted on the $x$-axis. Fold change 1047 in average sum read intensities, represented by a ratio between the LSD1-binding and 1048 LSD1-nonbinding sample in each comparison, is plotted on the $y$-axis. Each comparison, $1049 \mathrm{~A}-\mathrm{C}$, is represented by three panels. On top in each is the entire data set for that 1050 comparison, and below are two panels dividing the percentile rank score from 0-50 and 1051 from 50-100 with expanded $y$-axis limits to facilitate visualization of proteins enriched in 1052 the data set for the LSD1-binding form. Outlying proteins are labeled with their gene 1053 names. A red line indicates equal read intensities, and thus no enrichment, for the LSD11054 binding over the LSD1-nonbinding sample.

1056 Figure 7. BHC complex components HMG20A, HMG20B and PHF21A bind GFI1B in 1057 an LSD1-dependent manner. (A) BHC complex components are preferentially enriched 1058 in proximity labeling assays with fusion proteins competent for LSD1 binding. Biotin1059 modified proteins were purified from K562 cells inducibly expressing the BirA*:HA fusion 
1060 proteins shown. HMG20A, HMG20B, PHF21A, SMARCB1 and SMARCC1, as well as the

1061 BirA*:HA fusion proteins, were quantified in SAv-Sepharose pulldowns (SAv P) by

1062 western blot. Equivalent input for each protein was confirmed by western blot of whole

1063 cell lysates (Input). (B-D) GFI1B binding to HMG20A, HMG20B and PHF21A require

1064 GFI1B_LSD1 binding. COS7L cells were transfected with HA-tagged HMG20A (B),

1065 HMG20B (C), or PHF21A (D) and wild type (WT) or -P2A variants of GFI1B:flag. HMG20

1066 and PHF21A proteins and their binding partners were purified in $\alpha-\mathrm{HA}$ immune

1067 complexes. Co-precipitating GFI1B forms were detected in immune complexes by

1068 western blot using $\alpha$-flag antibody. Equivalent expression and precipitation were

1069 evaluated in WCLs and ICs, respectively. GFP served as a transfection control. (E)

1070 HMG20A and HMG20B bind LSD1. COS7L cells were transfected with HA-tagged

1071 HMG20A or HMG20B along with a V5-tagged LSD1. LSD1 and its binding partners were

1072 collected in $\alpha-V 5$ immune complexes (IC). Co-precipitating HMG20A and HMG20B were

1073 detected in immune complexes by western blot using $\alpha-\mathrm{HA}$ antibody. Equivalent

1074 expression and precipitation were evaluated in the whole cell lysates (WCL) and ICs, 1075 respectively.

1077 Figure 8. HMG20A and HMG20B are required for GFI1B-mediated cell fate changes

1078 in K562 cells. (A) HMG20A and HMG20B depletion in K562 cells. RNA (Top) and Protein

1079 (Bottom) were collected from K562 cells transduced with shRNA targeting HMG20A, 1080 HMG20B, HMG20A and HMG20B (HMG20A+B), or a content-matched scrambled 1081 control. HMG20A and HMG20B expression levels were evaluated by RT-qPCR and 1082 normalized to GUS. HMG20A, HMG20B, LSD1, and GFI1B:flag levels were determined 
1083 by western blot. Tubulin served as a loading control. (B-F) HMG20A/B depletion abolishes

1084 GFI1B-dependent cell fate determination in K562 cells. K562 cells were transduced with

1085 inducible shRNAs targeting HMG20A, HMG20B, HMG20A and B or a scrambled control.

1086 The shRNAs were induced with doxycycline, cells were infected with GFI1B:flag-

1087 expressing retrovirus and erythroid differentiation allowed to proceed. (B) Benzidine

1088 staining was quantified by counting positive cells in bright field microscopy while

1089 hemoglobinized cell pellets are shown above each condition shown in the bar graph. (C)

1090 GFI1B-dependent inhibition of TPA-induced CD61 expression requires HMG20A/B. K562

1091 cells overexpressing GFI1B and depleted of HMG20A, HMG20B, or HMG20A and B were

1092 treated with TPA and cell surface expression of CD61 quantified by flow cytometry.

1093 Histograms presented are representative of three independent experiments. (D-F) mRNA

1094 for globin genes $H B B$ and $H B Z(D)$, heme biosynthesis genes $A L A S 2$ and $F E C H(E)$ and

1095 GFI1B-repressed target genes GFI1B and MYB $(F)$ were quantified by RT-qPCR

1096 normalized to GUS reference gene. Results are expressed as mean \pm 2 SD. Statistical

1097 significance was determined by two-sided unpaired $t$-test; ${ }^{*}, p<0.05 ;{ }^{* *}, p<0.005$; ${ }^{* * * *}$, $1098 p<0.00005$.

1100 Figure 9. GSE1 is required for GFI1B-mediated cell fate changes in K562 cells. (A) 1101 GSE1 depletion in K562 cells. RNA was collected from K562 cells transduced with shRNA 1102 targeting GSE1 or a content-matched scrambled control. GSE1 expression levels were 1103 evaluated by RT-qPCR and normalized to GUS. (B-D) GSE1 depletion abolishes GFI1B1104 dependent cell fate determination in K562 cells. K562 cells were transduced with 1105 inducible shRNAs targeting GSE1 or a scrambled control. The shRNAs were induced with 
1106 doxycycline, cells infected with GFI1B:flag expressing retrovirus and erythroid

1107 differentiation allowed to proceed. (B) Benzidine staining was quantified by counting

1108 positive cells in bright field microscopy. (C-D) mRNA for globin and related genes $A H S P$,

$1109 H B A 1 / 2, H B Z$, and $H B B$; heme biosynthesis genes $A L A S 2$ and FECH (C); and GFI1B-

1110 repressed target genes GFI1B and CDKN1A (D) were quantified by RT-qPCR and

1111 normalized to GUS reference gene. Results are expressed as mean $\pm 2 S D$. (E) GFI1B-

1112 dependent inhibition of TPA-induced CD61 expression requires GSE1. K562 cells

1113 overexpressing GFI1B and depleted of GSE1 were treated with TPA and cell surface

1114 expression of CD61 quantified by flow cytometry compared to control cells. Histograms

1115 presented are representative of three independent experiments. Statistical significance

1116 was determined by two-sided unpaired $t$-test; ${ }^{* *}, p<0.005 ;{ }^{* * * *}, p<0.00005$.

1118 Figure 10. A working model for contributions of the BHC complex to GF1B-

1119 mediated repression. LSD1 serves as a bridge between GFI1B and other components

1120 of the BHC complex, including HDAC1/2, HMG20A, HMG20B, PHF21A, RCOR1, 1121 ZMYM2, ZNF217, and GSE1. Therefore, BHC complex recruitment is rendered LSD1

1122 dependent. A functional GFI1B transcriptional repression complex requires coincident

1123 recruitment of these factors, along with other partners brought to the promoter in an LSD1-

1124 independent manner by GFI1B or DNA binding proteins with which it collaborates.

1125 Dysfunction resulting from (1) an impaired, SNAG-dependent GFI1B_LSD1 recruitment

1126 mechanism (2) LSD1 depletion or intrinsic change that disrupts SNAG binding or (3)

1127 ineffective formation or operation of $\mathrm{BHC}$ complex components could each poison the 1128 GFI1B - LSD1 functional axis to create a permissive state for misexpression of GFI1B- 
1129 regulated genes. Only when each element is present and functioning properly (4) is the

1130 appropriate repressive outcome achieved. Trans-regulation by each component toward

1131 the others, or toward partners that engage GFI1B in a LSD1-independent manner, may

1132 also make important contributions to enabling and fine-tuning target gene expression.

1134 Table 1. LSD1-dependent components of GFI1B proximitomes. Shown are

1135 components of LSD1-containing transcription complexes represented in the GFI1B

1136 proximitome. The core and unique components of each complex are enumerated (top),

1137 and the number of core and unique components from these complexes found in each

1138 proximitome comparison is shown.

1139

1140 SUPPLEMENTARY MATERIALS

1141 Supplementary Data S1 File: McClellan et al Mass Spec Data.xlsx (1.6 MB)

1143 Table S1 Top 100 LSD1-dependent proximity partners for each comparison

1144

1145 Table S2 Oligonucleotides used in this study 


\section{Figure 1}

A
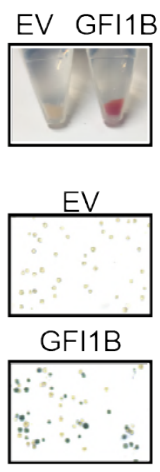

B

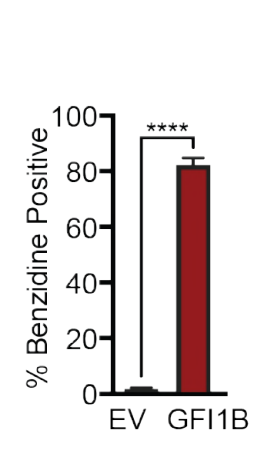

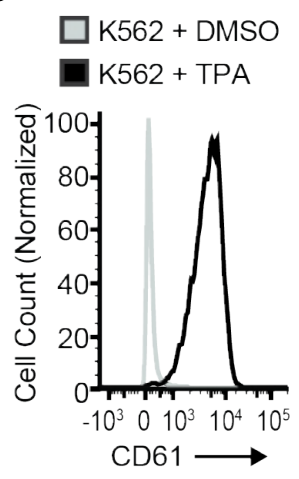

$\mathbf{F}$

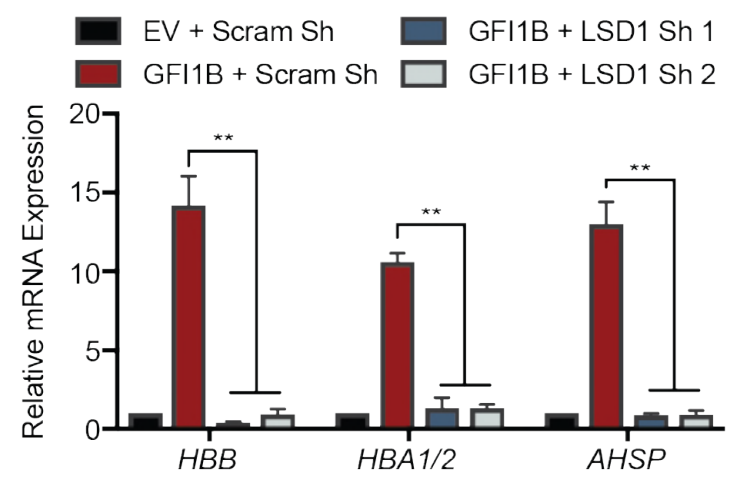

G

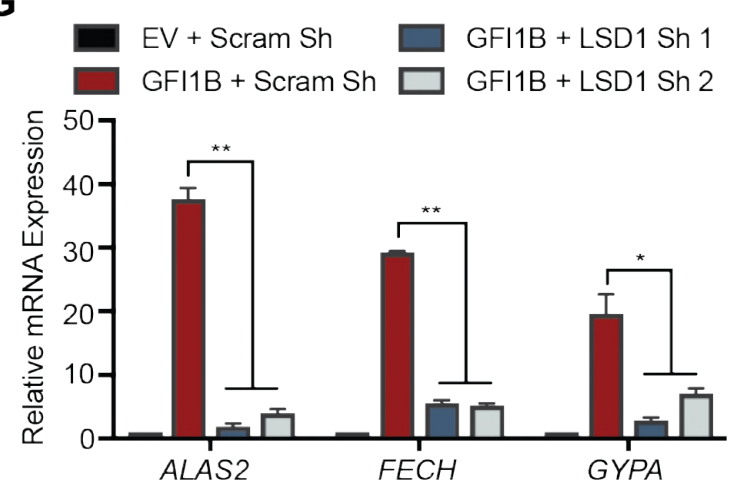

H

D
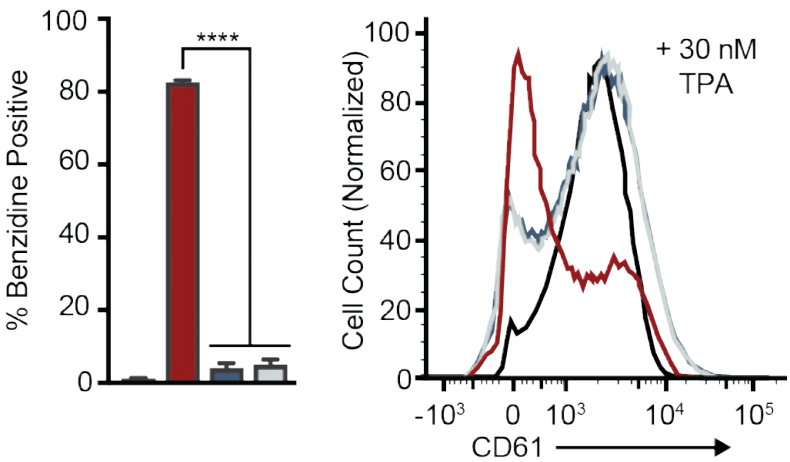

$E V+$ Scram Sh GFI1B + Scram Sh

$\square$ GFI1B + LSD1 Sh 1 GFI1B + LSD1 Sh 2
E

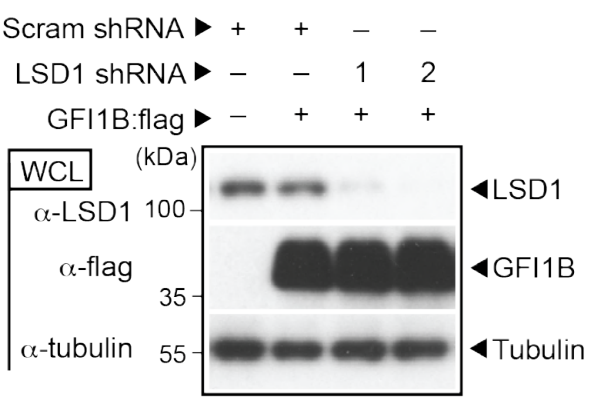

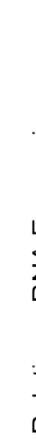

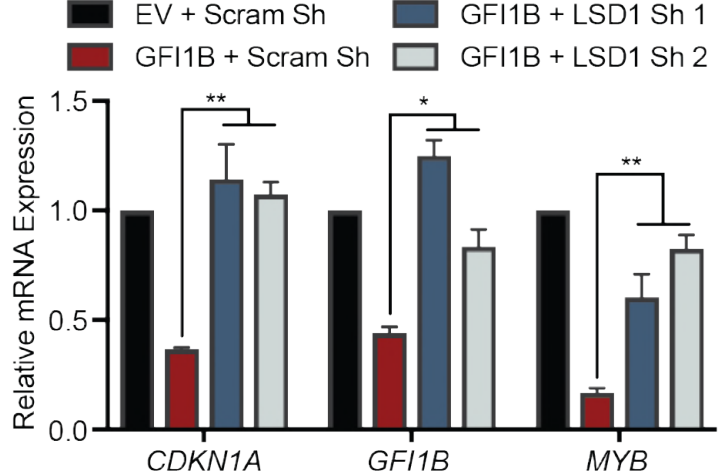

Figure 1. LSD1 depletion impedes GFI1B-mediated erythroid differentiation. (A) GFI1B overexpression promotes K562 cell hemoglobinization. Cells were infected with empty vector (EV) or virus expressing GFI1B. GFI1B-expressing cells become red and are benzidine positive. Benzidine positive cells are quantified as mean \pm SD from four biological replicates. (B) TPA triggers cell surface expression of CD61 in K562 cells. CD61 expression was quantified in vehiclevs. TPA-treated viable cells by flow cytometry. (C) LSD1 depletion and GFI1B overexpression in K562 cells. Small hairpin RNAs targeting LSD1 (LSD1 shRNA) or content-matched scramble control (Scram shRNA) were inducibly transcribed in K562 cells followed by infection at day +3 with GFI1B:flag-expressing virus or EV. LSD1 and GFI1B were quantified by western blot of whole cell lysates (WCL). Tubulin served as a loading control. (D) LSD1 depletion blocks GFI1B- 
mediated K562 cell hemoglobinization. (E) LSD1 depletion enables TPA-induced CD61 expression in the context of GFI1B overexpression. (F-G) LSD1 depletion impairs expression of erythroid fate genes in response to GFI1B overexpression. Alpha- and Beta-globins (HBA1/2 and $H B B)$, alpha globin stabilizing protein $(A H S P)$, aminolevulinic acid synthase $(A L A S) 2$, ferrochelatase $(F E C H)$ and glycophorin $(G Y P) A$ expression are shown. $(H)$ LSD1 depletion restores expression of GFI1B-repressed target genes. CDKN1A, GFI1B and MYB expression are shown. Gene expression is quantified by RT-qPCR normalized to GUS. Results are expressed as mean $\pm 2 S D$ from two independent experiments performed in triplicate. ${ }^{*} p<0.05$; ${ }^{* *} p<0.005$; ${ }^{* * *} p<0.0005,{ }^{* * * *} p<0.00005$. 


\section{Figure 2}

A

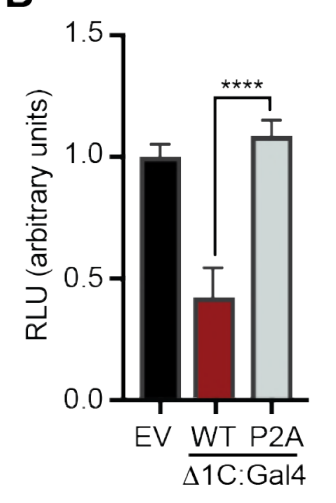

C

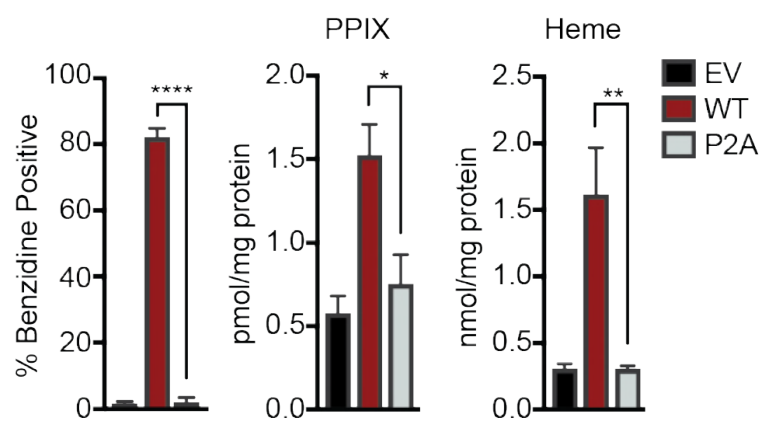

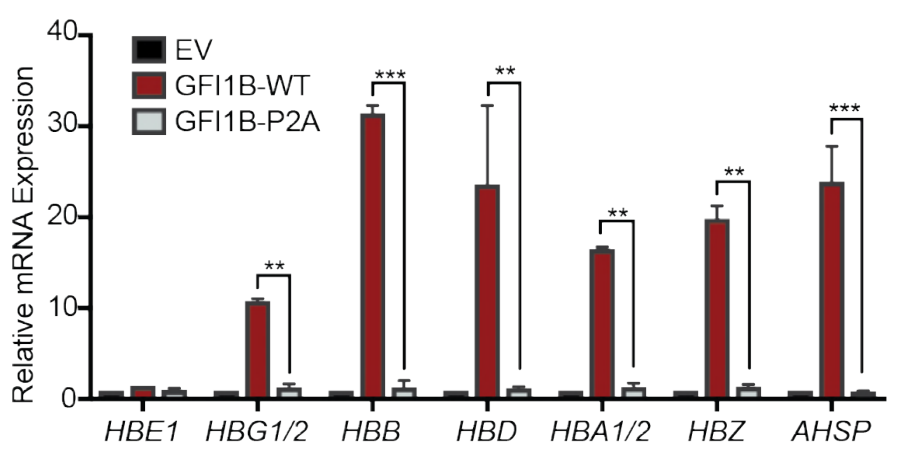

E

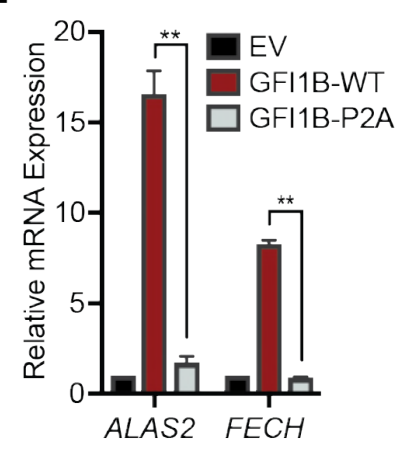

$\mathbf{F}$

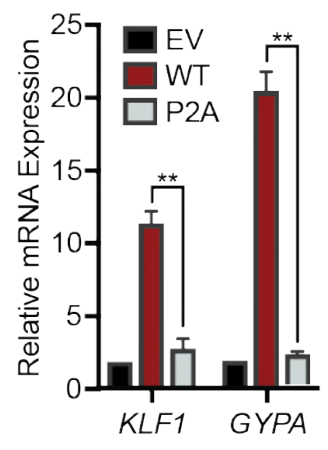

H

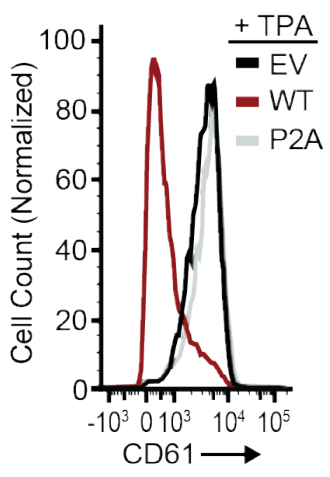

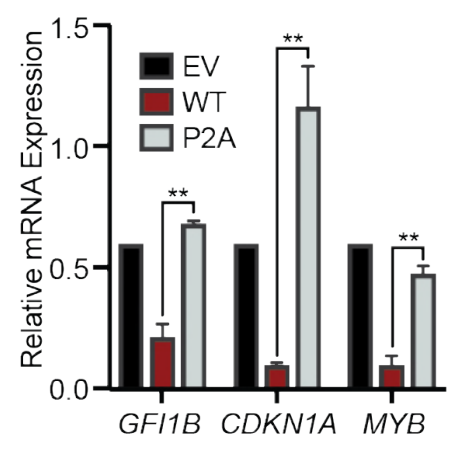

Figure 2. LSD1 binding is required for GFI1B-regulated cell fate decisions in K562 cells. (A) P2A substitution in GFI1B impairs GFI1B-LSD1 binding. K562 cells were transduced with constructs expressing wild type GFI1B, its P2A variant or empty vector (EV) as shown. GFI1B forms were immune purified with $\alpha$-flag (M2) antibody and Protein-G Sepharose. LSD1 was quantified in immune complexes (IC) by western blot. Equal expression (LSD1 and GFI1B) and precipitation (GFI1B) were evaluated by western blot of whole cell lysates (WCL) and ICs, respectively. Tubulin was used to confirm equal gel loading. (B) P2A substitution abolishes transcriptional repression by GFI1B. 293-T-Rex-5xGal-luciferase cells were transfected with WT or P2A variants of GFI1B- $\Delta 1 \mathrm{C}$ :Gal4 (left) or SNAG:Gal4 (right) fusion proteins. Firefly luciferase was measured and normalized to constitutively expressed, co-transfected Renilla luciferase. 
Reporter activity is expressed as mean $\pm S D$ from two experiments performed in triplicate. (C) P2A substitution impairs GFI1B-mediated hemoglobinization. Benzidine staining was quantified as in 1A. Protoporphyrin IX (PPIX) and heme were quantified by UPLC in K562 cells transduced with EV, GFI1B-WT or GFI1B-P2A. (D-F) P2A substitution impairs expression of erythroid fate genes in K562 cells. Expression of $\beta$-globin cluster genes, AHSP, ALAS2, FECH, GYPA and Kruppellike factor $(K L F) 1$ are shown. (G) P2A substitution abolishes GFI1B-mediated suppression of TPA-induced CD61 expression. Cell surface expression of CD61 in TPA treated cells expressing wild type GFI1B (WT) or its P2A variant was determined by flow cytometry. (H) P2A substitution reverses repression of GFI1B target genes GFI1B, CDKN1A and MYB. Results expressed as mean \pm 2 SD. ${ }^{*}, p<0.05 ;{ }^{* *}, p<0.005 ;{ }^{* * *}, p<0.0005 ;{ }^{* * *}, p<0.00005$. 


\section{Figure 3}

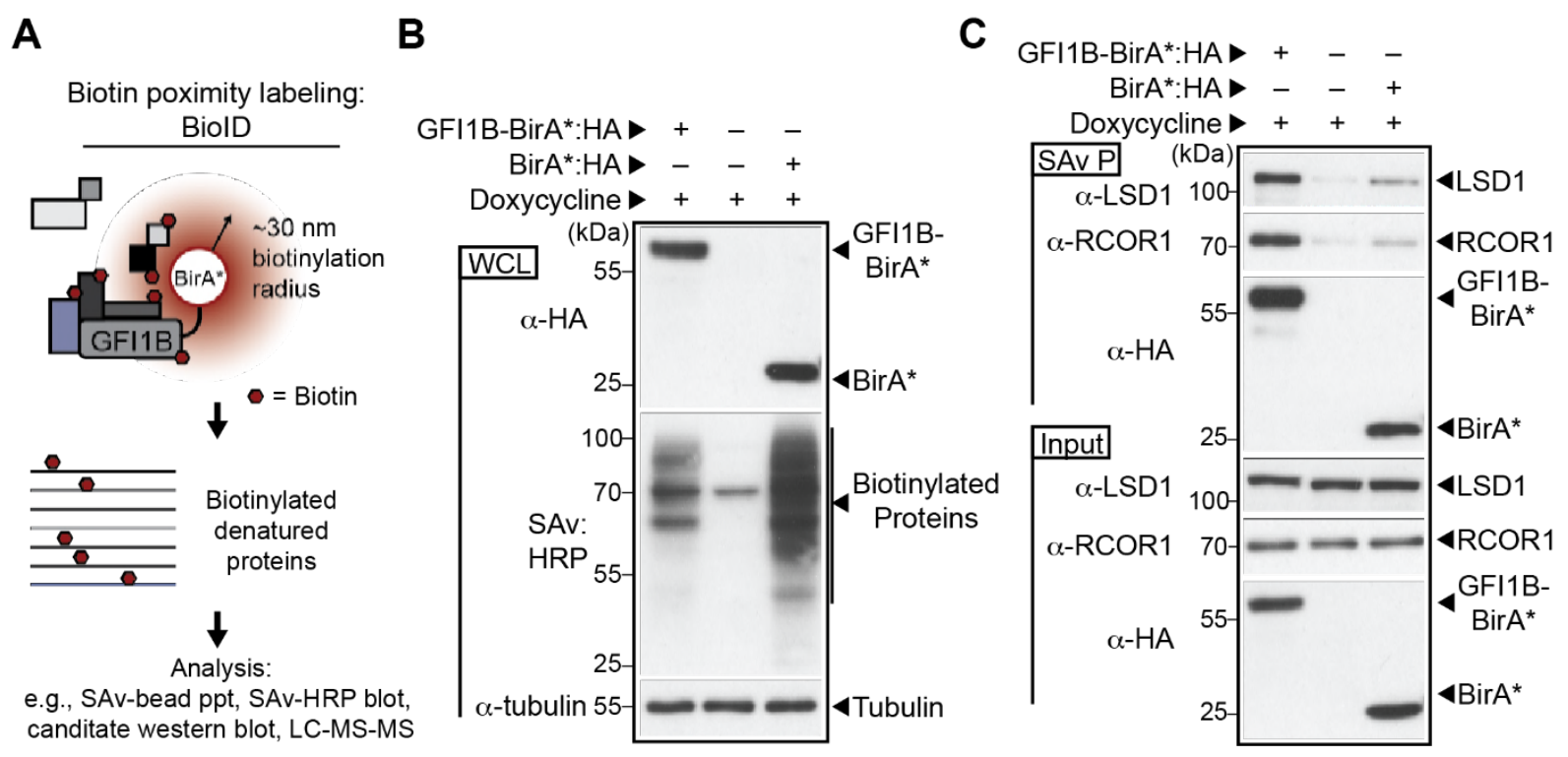

D

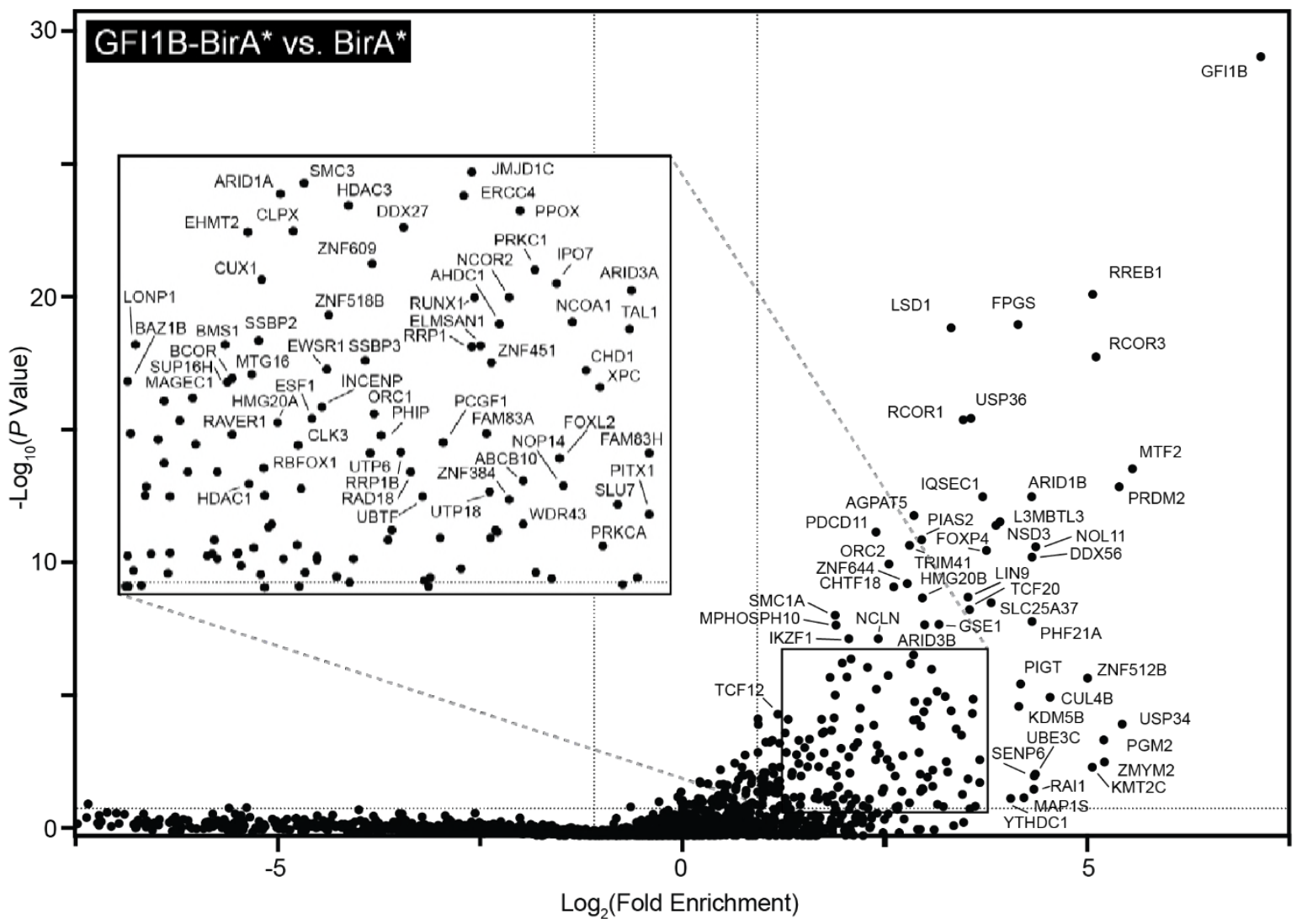

Figure 3. GFIB-BirA* defines a GFI1B proximitome. (A) Schematic representation of strategy for biotin modification of GFI1B partners. GFI1B is fused to the promiscuous biotin ligase with an 
HA epitope tag, BirA*:HA to form GFI1B-BirA*:HA. GFI1B partners are spontaneously biotinylated due to their proximity to BirA*:HA anchored to GFI1B in cis. Biotinylated products are captured on streptavidin (SAv)-conjugated beads for downstream analysis via candidate-based or screening approaches. (B) BirA*:HA and GFI1B-BirA*:HA fusion biotinylate diverse targets in K562 cells. Cells were transduced with constructs inducibly expressing GFI1B-BirA*:HA, BirA*:HA or EV, treated with doxycycline and harvested. GFI1B-BirA*:HA and BirA*:HA expression was confirmed by $\alpha-H A$ western blot and biotinylated proteins detected by SAv:HRP. Tubulin served as a loading control. (C) GFI1B interacting protein, LSD1 and RCOR1, are enriched among proteins biotinylated by GFI1B-BirA*:HA compared to BirA* or EV., Biotin-modified proteins were collected on SAv-Sepharose. LSD1, RCOR1 and the BirA* fusion proteins themselves were quantified among purified proteins ( $S A v P$ ) by western blot. Equal expression was confirmed by western blot in whole cell lysates (input). (D) GFI1B-BirA*:HA identifies members of a GFI1B proximitome. Factors enriched among biotinylated and SAv-Sepharose purified proteins modified by GFI1BirA*:HA (test) compared to BirA*:HA (control). Fold enrichment is plotted logarithmically as a ratio of average sum read intensities between test and control samples against their $p$-values calculated from their 20 closest ranked neighbors in the data set as described in Materials and Methods. Individual proteins are labeled with their respective gene names. Inset shows an expanded view of the data contained in the boxed area. 


\section{Figure 4}

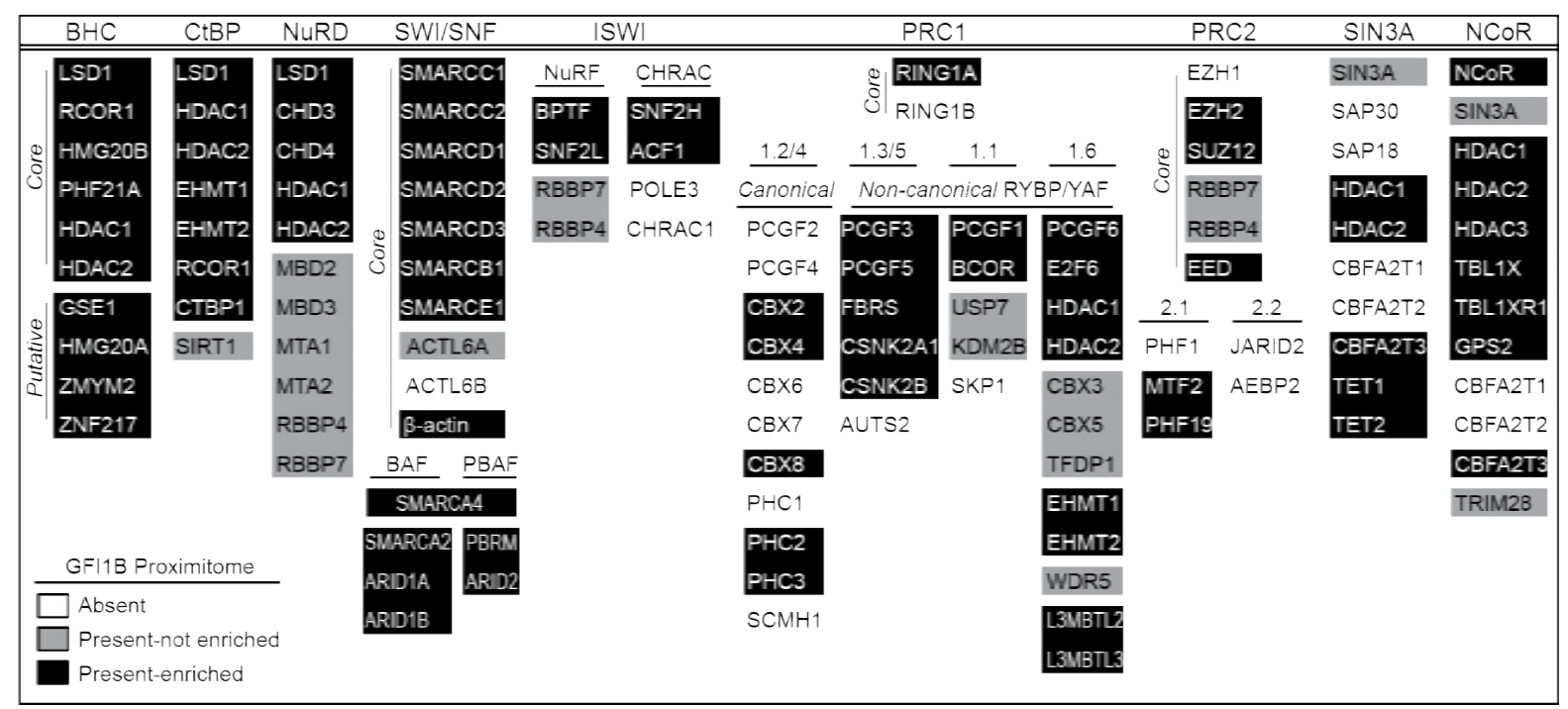

Figure 4. Components of transcriptional repression and chromatin remodeling complexes identified by GFI1B proximity labeling. A roster of transcriptionally relevant complexes and their component members are shown. Proteins are listed according to HUGO gene nomenclature. With respect to the GFI1B proximitome (GFI1B-BirA*:HA vs. BirA*:HA), proteins present and enriched-white letters on black background. Those present by not significantly enriched ( $p$ $<0.05$ ) - black letters on gray background. Not present (average sum read intensity below that for EV)—black letters on white background. Where appropriate, complexes are subdivided into core and putative members, and/or with canonical vs. non-canonical designations. 


\section{Figure 5}

A

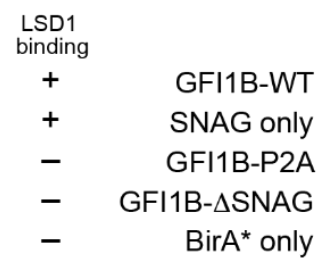

B

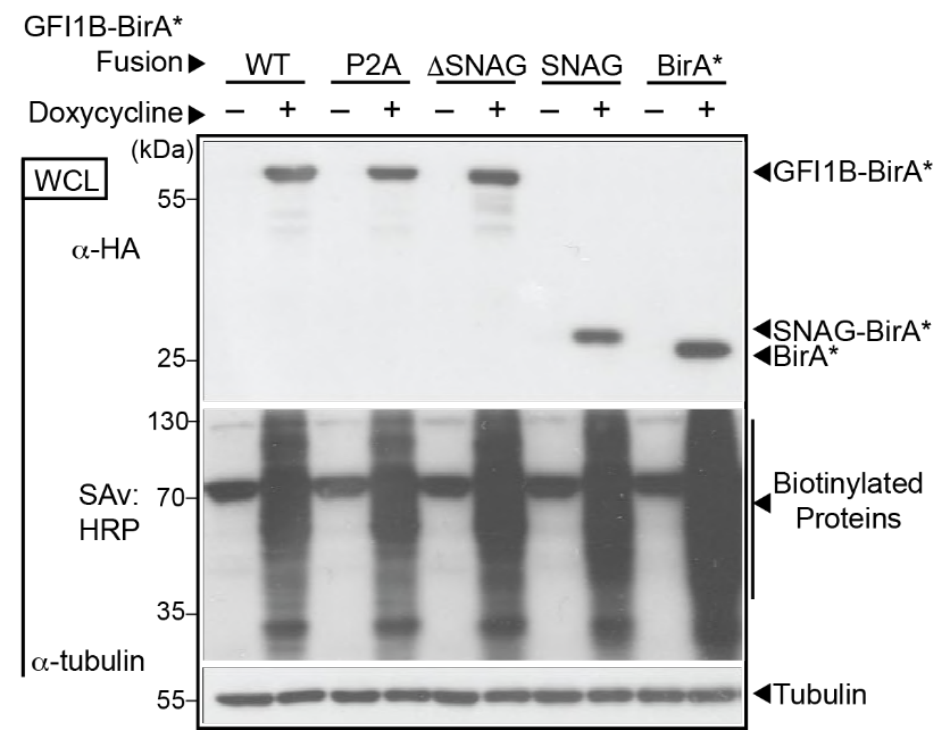

C

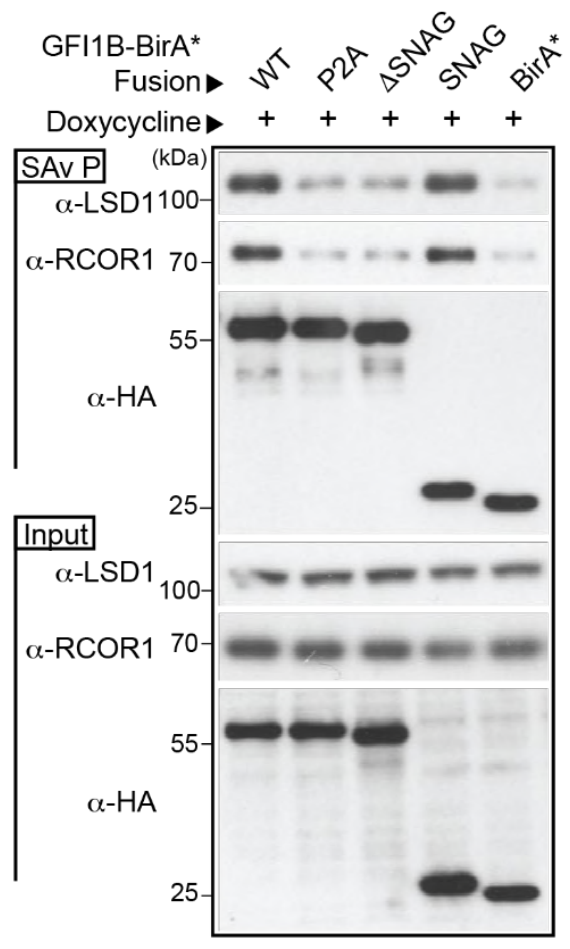

Figure 5. LSD1 non-binding variants of GFI1B-BirA* fail to biotinylate LSD1 and RCOR1. (A) Graphical representation of GFI1B-BirA*:HA fusion proteins employed in experiments. Each fusion protein is comprised of the GFI1B regions shown in frame with the Bir $A^{*}$ expression cassette and a C-terminal HA epitope tag to insure equivalent expression of all forms. The BirA*:HA construct lacks GFI1B structures and instead begins with an ATG. Wild type (WT) GFI1B is shown. The SNAG and zinc finger $(\mathrm{ZnF})$ domains (1-6) of GFI1B are shown as black boxes. A thin line represents the GFI1B linker and connections between ZnFs. The proline to alanine substitution (P2A) in the GFI1B SNAG domain is indicated with an *. $\triangle$ SNAG represents deletion of the SNAG domain but preservation of the remaining GFI1B primary structure. (B) Expression of GFI1B-BirA*:HA forms and proteome-wide biotin modification in K562 cells. K562 cells were transduced with lentivirus to inducibly express GFI1B-BirA*:HA forms shown and stable isolates selected as polyclonal populations. Doxycycline-inducible expression of each fusion protein was confirmed by western blot against the common HA epitope tag and biotinylating activity confirmed proteome-wide by SAv:HRP detection of transblotted total cellular protein. Tubulin served as a loading control. (C) LSD1 and RCOR1 are enriched among biotinylated proteins generated by BirA*:HA fusions competent for LSD1 binding. Biotin-modified proteins from K562 cells transduced with BirA*:HA fusion proteins shown were purified from whole cell extracts (SAv $P$ ), fractionated by SDS-PAGE and subjected to western blot with $\alpha$-LSD1, $\alpha$-RCOR1 and $\alpha-H A$ antibodies. Equivalent expression of each protein in whole cell lysates (Input) was confirmed by western blot with these same antibodies. 


\section{Figure 6}

\section{A}
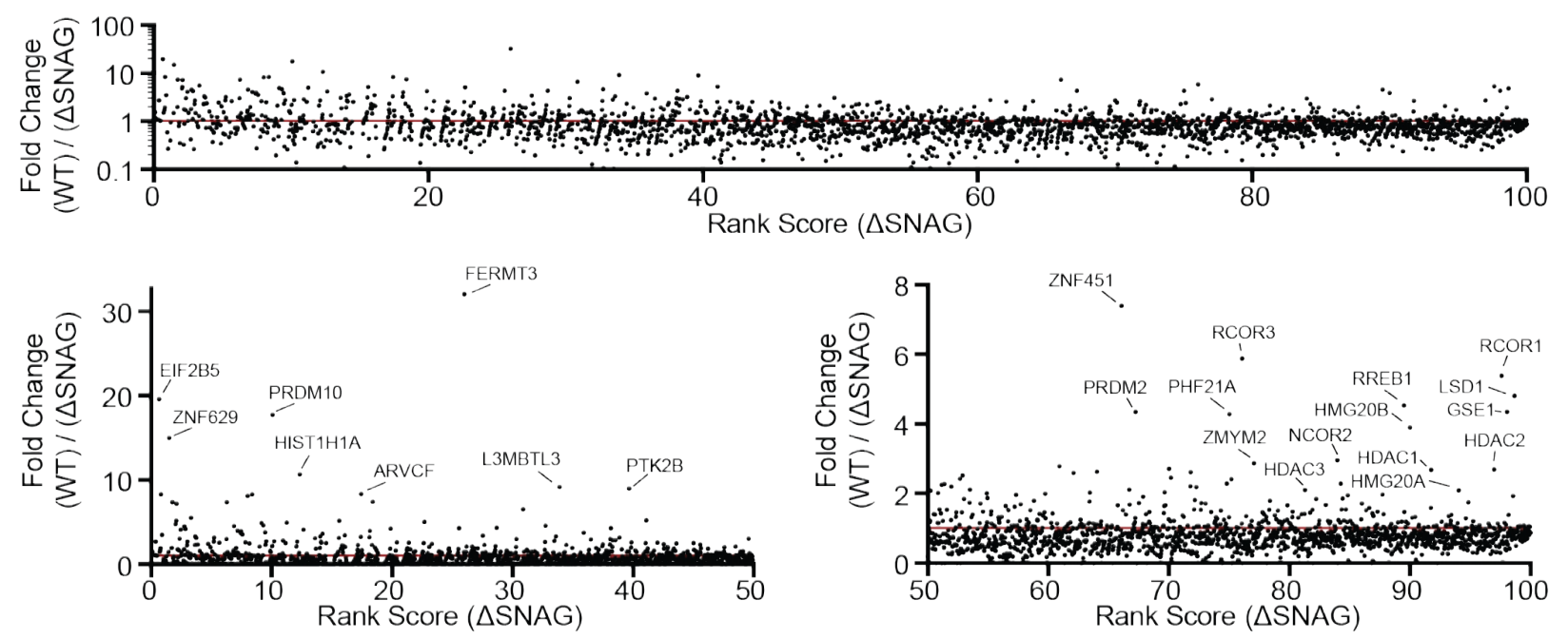

B
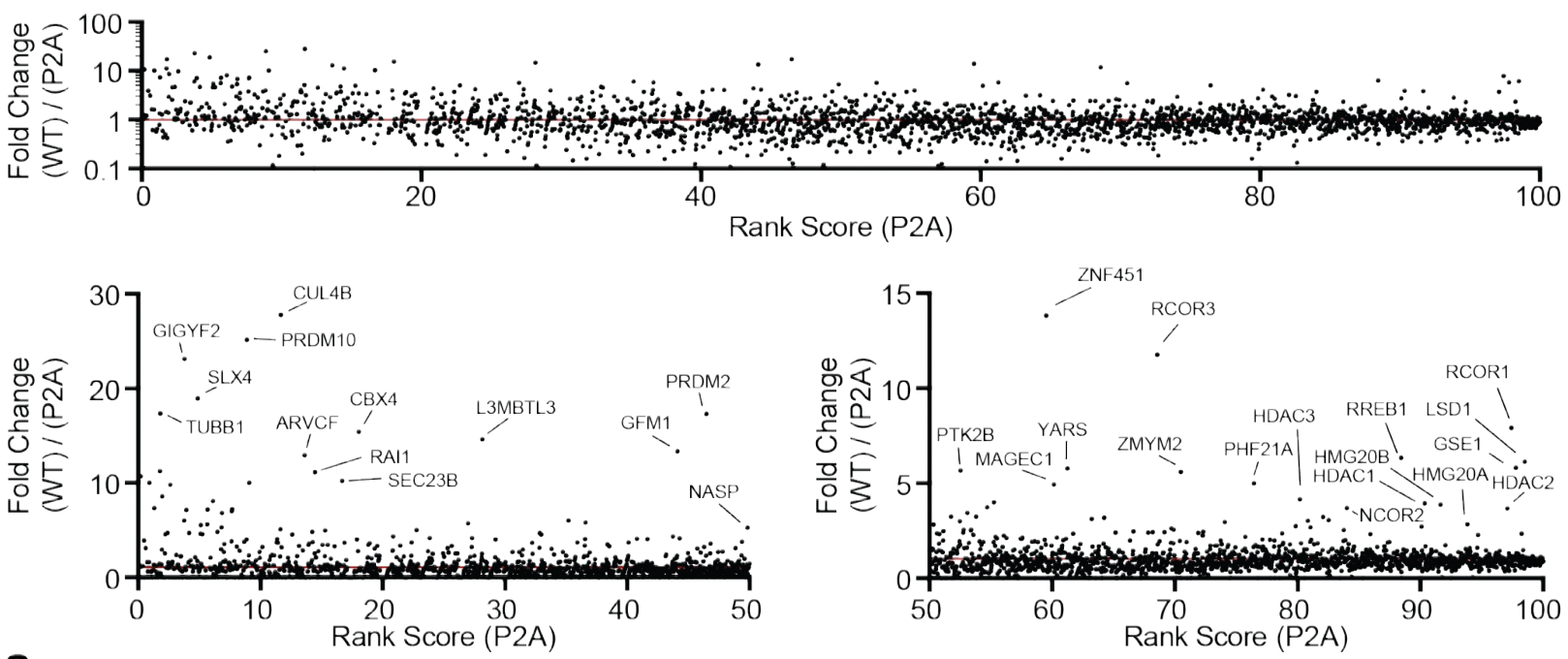

C
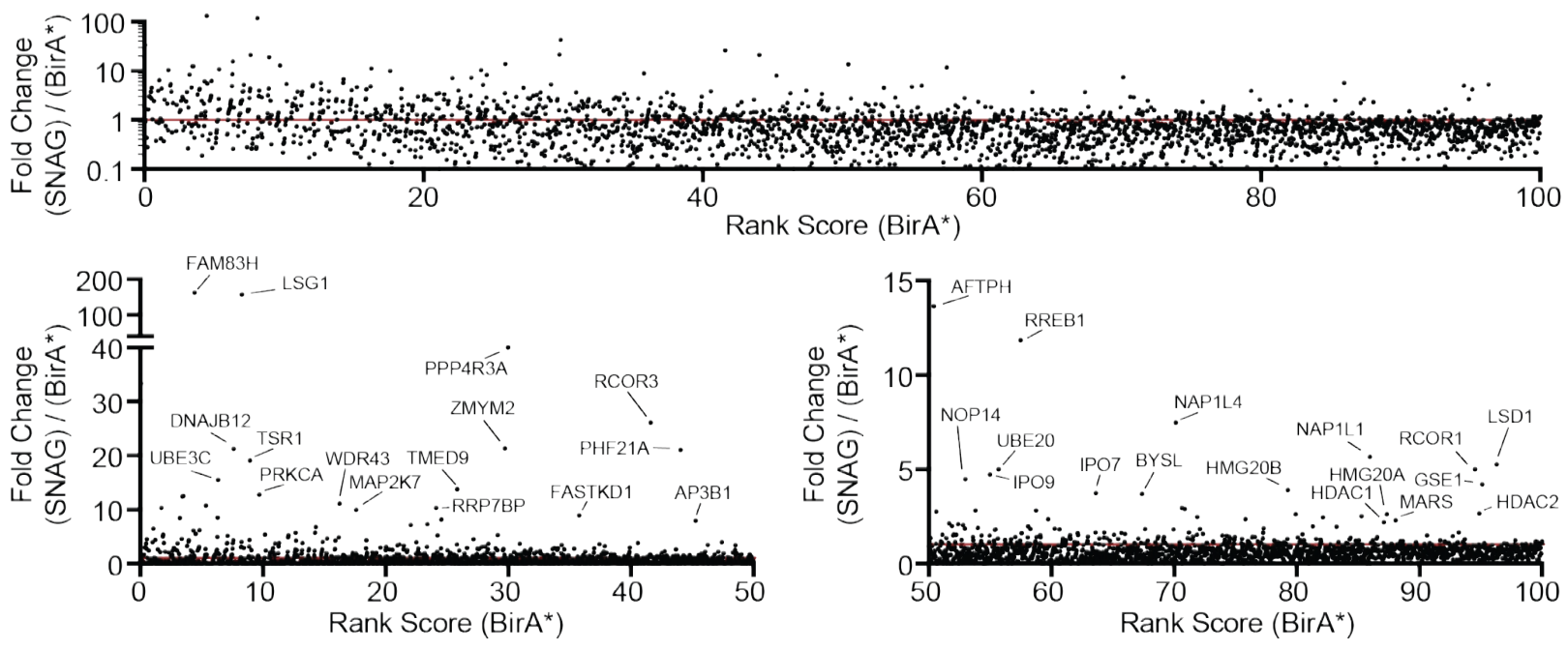
Figure 6. GFI1B recruits the BHC complex in an LSD1-depend manner via the SNAG domain. K562 cells stably and inducibly expressing the BirA*:HA fusion proteins shown in 5A were deployed to define GFI1B proximity partners whose recruitment is LSD1-dependent. Fusion protein expression was induced by doxycycline addition and biotinylation permitted in situ. Biotinmodified proteins were captured on SAv-Sepharose and analyzed by LC-MS-MS as described in Materials and Methods. (A-C) Dot plots are shown comparing fold change in average sum read intensity among fusion proteins with intact SNAG domains and capable of LSD1-binding (GFI1BBirA*:HA and SNAG-BirA*:HA) to those with disrupted or absent SNAG domains and deficient in LSD1 binding (GFI1B-P2A-BirA*:HA, GFI1B- $\triangle S N A G-B i r A^{*}: H A$, and BirA*:HA). For each comparison (A-GFI1B-BirA*:HA vs. GFI1B- $\triangle$ SNAG-BirA*:HA, B-GFI1B-BirA* vs. GFI1B-P2ABirA*:HA, and $C-S N A G-B i r A^{*}: H A$ vs. BirA*:HA), average sum read intensities were ranked according to the LSD1-nonbinding sample and plotted on the $x$-axis. Fold change in average sum read intensities, represented by a ratio between the LSD1-binding and LSD1-nonbinding sample in each comparison, is plotted on the $y$-axis. Each comparison, A-C, is represented by three panels. On top in each is the entire data set for that comparison, and below are two panels dividing the percentile rank score from 0-50 and from 50-100 with expanded $y$-axis limits to facilitate visualization of proteins enriched in the data set for the LSD1-binding form. Outlying proteins are labeled with their gene names. A red line indicates equal read intensities, and thus no enrichment, for the LSD1-binding over the LSD1-nonbinding sample. 


\section{Figure 7}

A

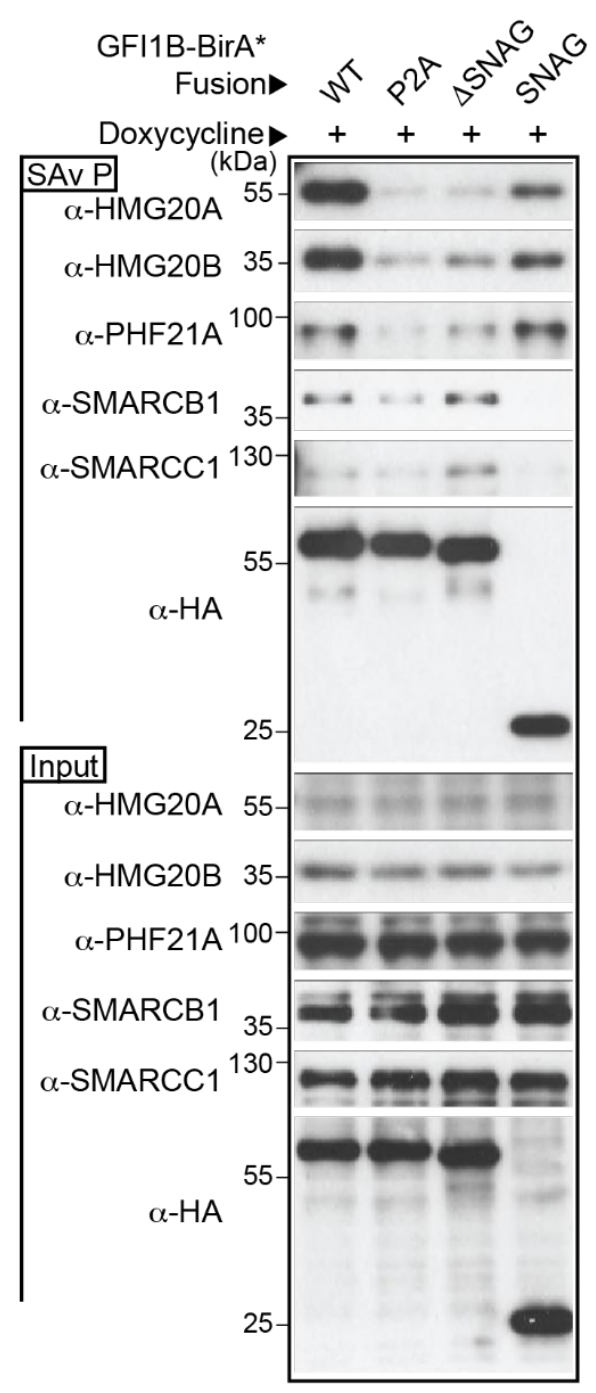

B

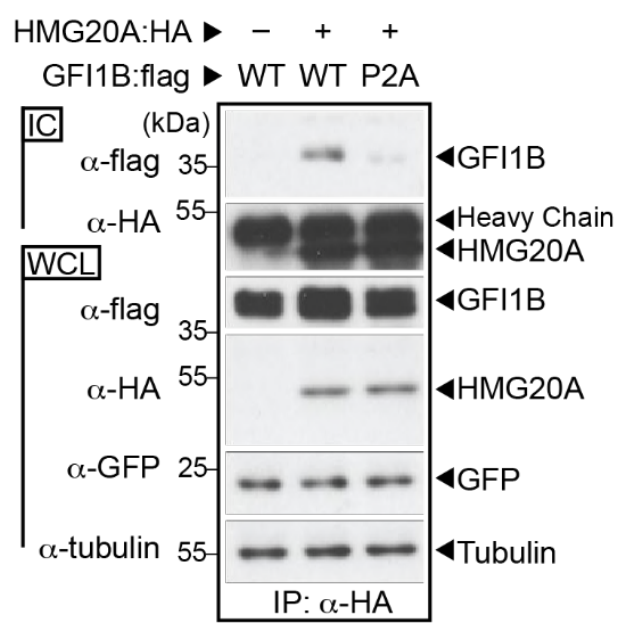

C

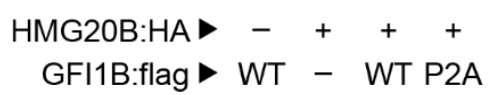

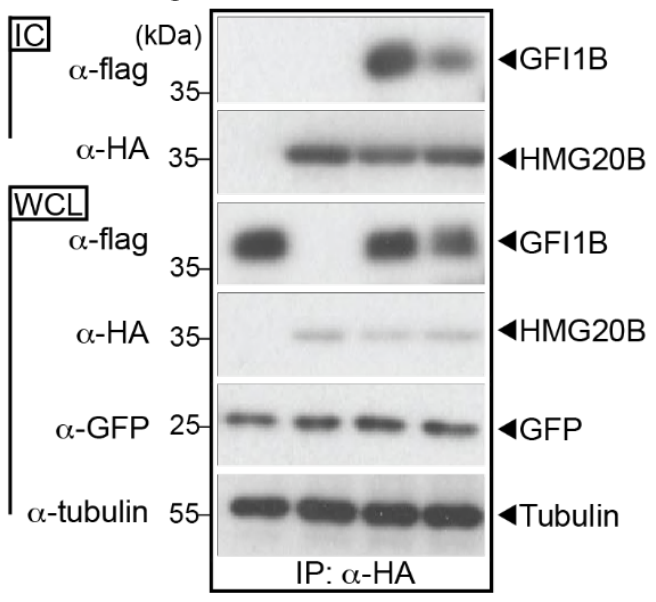

D

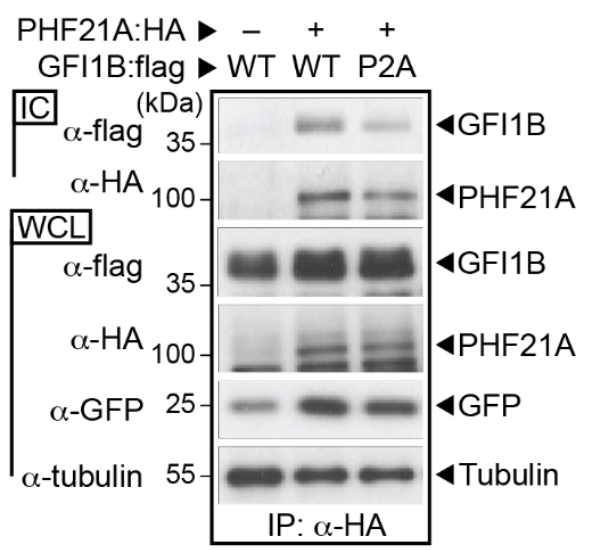

E

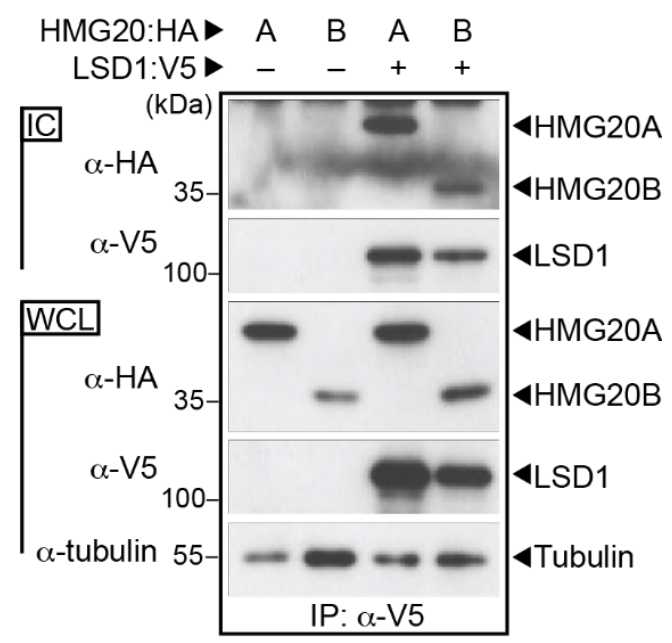


Figure 7. BHC complex components HMG20A, HMG20B and PHF21A bind GFI1B in an LSD1-dependent manner. (A) BHC complex components are preferentially enriched in proximity labeling assays with fusion proteins competent for LSD1 binding. Biotin-modified proteins were purified from K562 cells inducibly expressing the BirA*:HA fusion proteins shown. HMG20A, HMG20B, PHF21A, SMARCB1 and SMARCC1, as well as the BirA*:HA fusion proteins, were quantified in $S A v$-Sepharose pulldowns ( $S A v P)$ by western blot. Equivalent input for each protein was confirmed by western blot of whole cell lysates (Input). (B-D) GFI1B binding to HMG20A, HMG20B and PHF21A require GFI1B - LSD1 binding. COS7L cells were transfected with HAtagged HMG20A (B), HMG20B (C), or PHF21A (D) and wild type (WT) or -P2A variants of GFI1B:flag. HMG20 and PHF21A proteins and their binding partners were purified in $\alpha-H A$ immune complexes. Co-precipitating GFI1B forms were detected in immune complexes by western blot using $\alpha$-flag antibody. Equivalent expression and precipitation were evaluated in WCLs and ICs, respectively. GFP served as a transfection control. (E) HMG20A and HMG20B bind LSD1. COS7L cells were transfected with HA-tagged HMG20A or HMG20B along with a V5tagged LSD1. LSD1 and its binding partners were collected in $\alpha-V 5$ immune complexes (IC). Coprecipitating HMG20A and HMG20B were detected in immune complexes by western blot using $\alpha-\mathrm{HA}$ antibody. Equivalent expression and precipitation were evaluated in the whole cell lysates (WCL) and ICs, respectively. 


\section{Figure 8}

A
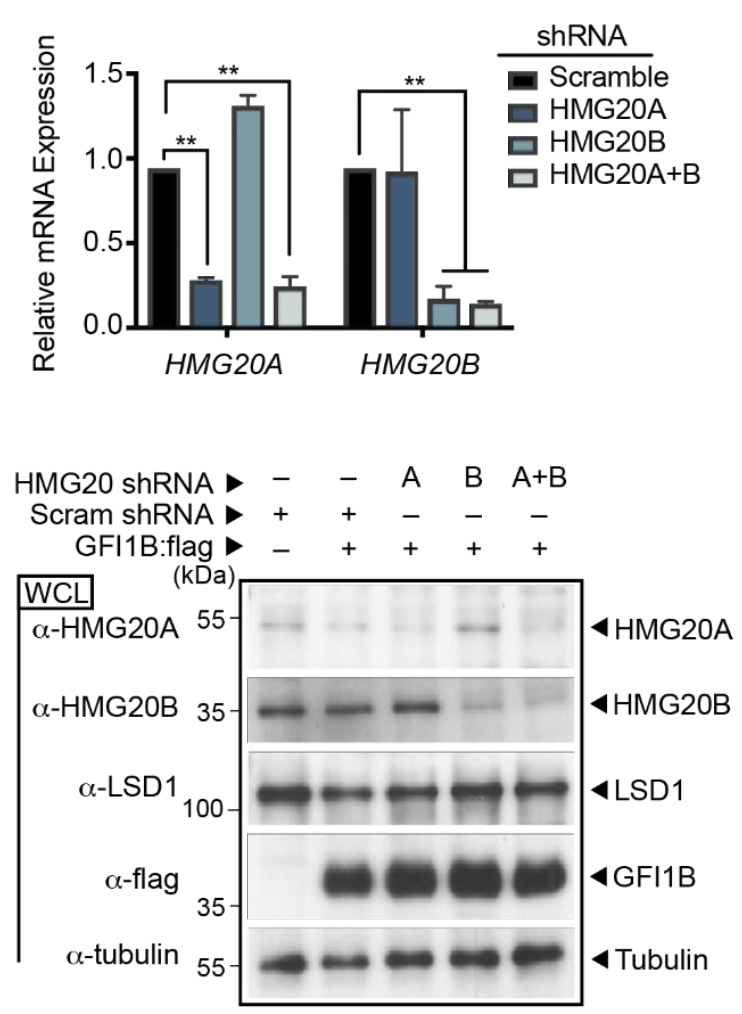

B

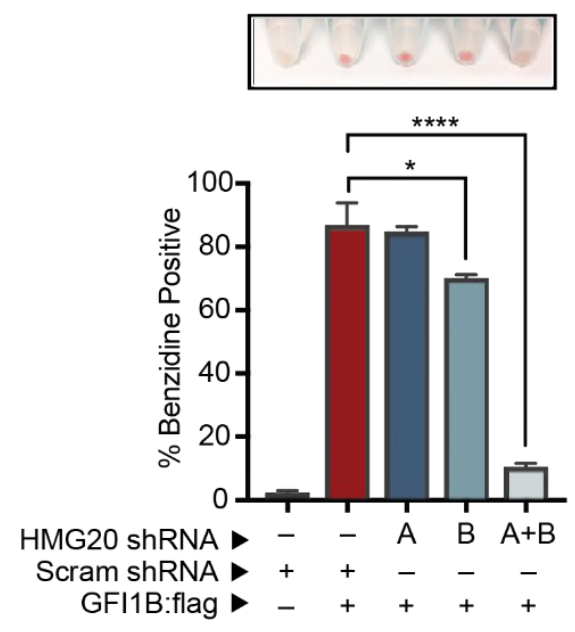

C

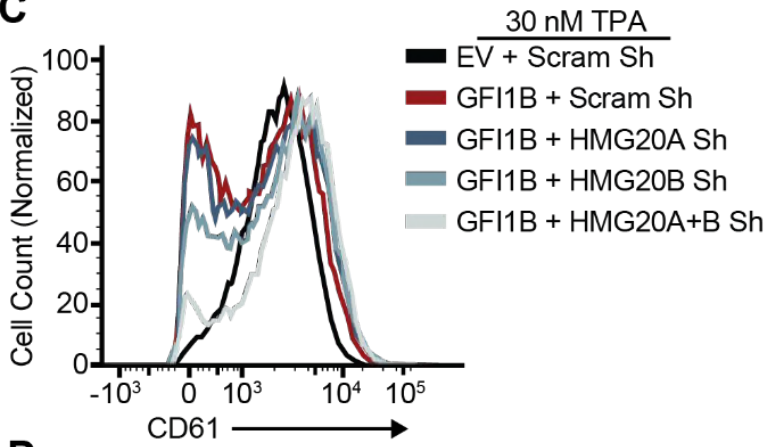

D

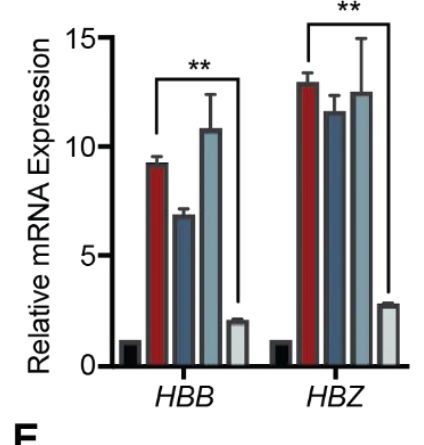

EV + Scram Sh

GFI1B + Scram Sh

GFI1B + HMG20A Sh

GFI1B + HMG20B Sh

$\square$ GFI1B + HMG20A+B Sh

E

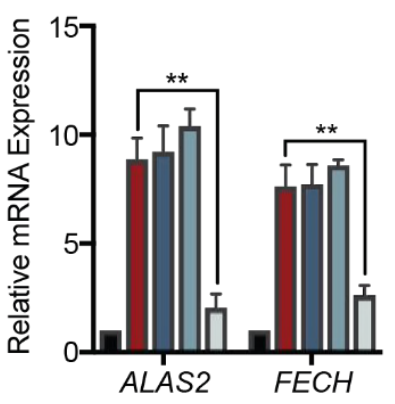

EV + Scram Sh

GFI1B + Scram Sh

GFI1B + HMG20A Sh

$\square$ GFI1B + HMG20B Sh

$\square$ GFI1B + HMG20A+B Sh

F

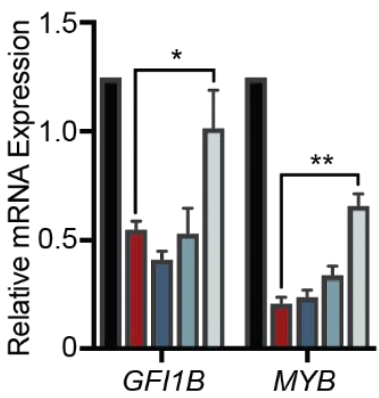

EV + Scram Sh GFI1B + Scram Sh $\square$ GFI1B + HMG20A Sh $\square$ GFI1B + HMG20B Sh $\square$ GFI1B + HMG2OA+B Sh

Figure 8. HMG20A and HMG20B are required for GFI1B-mediated cell fate changes in K562 cells. (A) HMG20A and HMG20B depletion in K562 cells. RNA (Top) and Protein (Bottom) were collected from K562 cells transduced with shRNA targeting HMG20A, HMG20B, HMG20A and HMG20B (HMG20A+B), or a content-matched scrambled control. HMG20A and HMG20B expression levels were evaluated by RT-qPCR and 
normalized to GUS. HMG20A, HMG20B, LSD1, and GFI1B:flag levels were determined by western blot. Tubulin served as a loading control. (B-F) HMG20A/B depletion abolishes GFI1B-dependent cell fate determination in K562 cells. K562 cells were transduced with inducible shRNAs targeting HMG20A, HMG20B, HMG20A and B or a scrambled control. The shRNAs were induced with doxycycline, cells were infected with GFI1B:flagexpressing retrovirus and erythroid differentiation allowed to proceed. (B) Benzidine staining was quantified by counting positive cells in bright field microscopy while hemoglobinized cell pellets are shown above each condition shown in the bar graph. (C) GFI1B-dependent inhibition of TPA-induced CD61 expression requires HMG20A/B. K562 cells overexpressing GFI1B and depleted of HMG20A, HMG20B, or HMG20A and B were treated with TPA and cell surface expression of CD61 quantified by flow cytometry. Histograms presented are representative of three independent experiments. (D-F) mRNA for globin genes $H B B$ and $H B Z$ (D), heme biosynthesis genes $A L A S 2$ and $F E C H(E)$ and GFI1B-repressed target genes GFI1B and MYB (F) were quantified by RT-qPCR normalized to GUS reference gene. Results are expressed as mean $\pm 2 S D$. Statistical significance was determined by two-sided unpaired $t$-test; ${ }^{*}, p<0.05 ;{ }^{* *}, p<0.005 ;{ }^{* * * *}$, $p<0.00005$. 


\section{Figure 9}

A

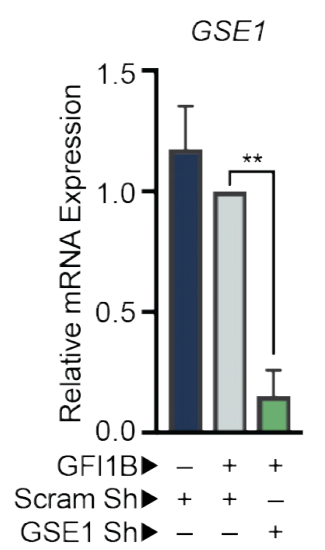

B

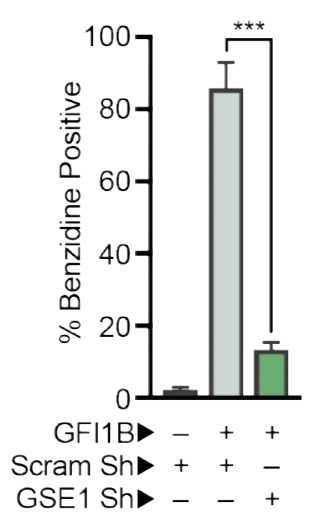

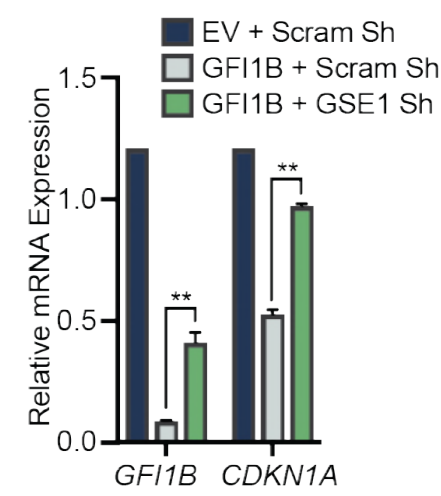

E

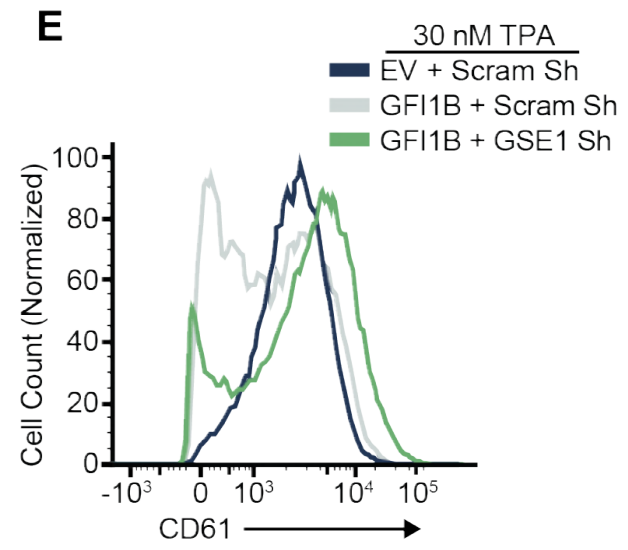

Figure 9. GSE1 is required for GFI1B-mediated cell fate changes in K562 cells. (A) GSE1 depletion in K562 cells. RNA was collected from K562 cells transduced with shRNA targeting GSE1 or a content-matched scrambled control. GSE1 expression levels were evaluated by RTqPCR and normalized to GUS. (B-D) GSE1 depletion abolishes GFI1B-dependent cell fate determination in K562 cells. K562 cells were transduced with inducible shRNAs targeting GSE1 or a scrambled control. The shRNAs were induced with doxycycline, cells infected with GFI1B:flag expressing retrovirus and erythroid differentiation allowed to proceed. (B) Benzidine staining was quantified by counting positive cells in bright field microscopy. (C-D) mRNA for globin and related genes $A H S P, H B A 1 / 2, H B Z$, and $H B B$; heme biosynthesis genes ALAS2 and $F E C H(C)$; and GFI1B-repressed target genes GFI1B and CDKN1A (D) were quantified by RT-qPCR and normalized to GUS reference gene. Results are expressed as mean $\pm 2 S D$. (E) GFI1B-dependent inhibition of TPA-induced CD61 expression requires GSE1. K562 cells overexpressing GFI1B and depleted of GSE1 were treated with TPA and cell surface expression of CD61 quantified by flow cytometry compared to control cells. Histograms presented are representative of three independent experiments. Statistical significance was determined by two-sided unpaired $t$-test; ${ }^{* *}, p<0.005 ;{ }^{* * * *}, p<0.00005$. 


\section{Figure 10}

(1)

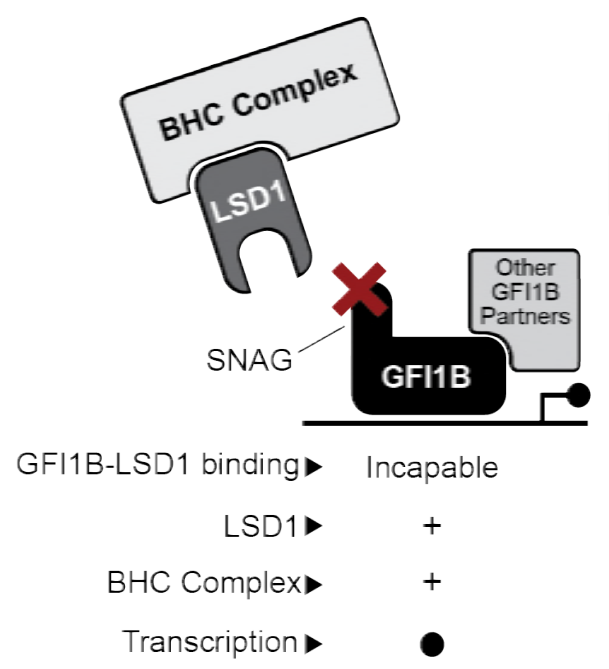

(2)

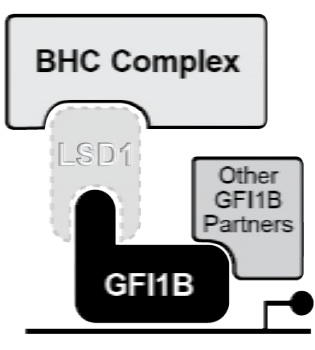

$\mathrm{n} / \mathrm{a}$

$-$
(3)

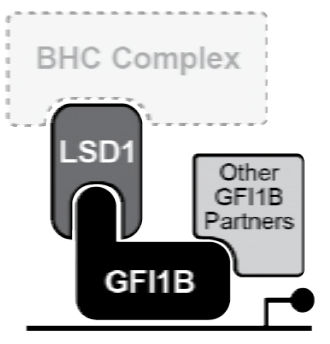

Capable

$+$

$-$

Figure 10. A working model for contributions of the BHC complex to GF1B-mediated repression. LSD1 serves as a bridge between GFI1B and other components of the $B H C$ complex, including HDAC1/2, HMG20A, HMG20B, PHF21A, RCOR1, ZMYM2, ZNF217, and GSE1. Therefore, BHC complex recruitment is rendered LSD1 dependent. A functional GFI1B transcriptional repression complex requires coincident recruitment of these factors, along with other partners brought to the promoter in an LSD1-independent manner by GFI1B or DNA binding proteins with which it collaborates. Dysfunction resulting from (1) an impaired, SNAG-dependent GFI1B - LSD1 recruitment mechanism (2) LSD1 depletion or intrinsic change that disrupts SNAG binding or (3) ineffective formation or operation of $\mathrm{BHC}$ complex components could each poison the GFI1B-LSD1 functional axis to create a permissive state for misexpression of GFI1Bregulated genes. Only when each element is present and functioning properly (4) is the appropriate repressive outcome achieved. Trans-regulation by each component toward the others, or toward partners that engage GFI1B in a LSD1-independent manner, may also make important contributions to enabling and fine-tuning target gene expression. 
Table 1 GFI1B LSD1-dependent proximitomes compared

\begin{tabular}{|c|c|c|c|c|c|c|}
\hline \multirow{2}{*}{$\begin{array}{l}\text { Complexes \& } \\
\text { Composition }\end{array}$} & \multicolumn{2}{|c|}{$\mathrm{BHC}$} & \multicolumn{2}{|c|}{ CtBP } & \multicolumn{2}{|c|}{ NuRD } \\
\hline & Total & Unique & Total & Unique & Total & Unique \\
\hline & 10 & 6 & 8 & 4 & 11 & 8 \\
\hline $\begin{array}{c}\text { Proximitomes } \\
\text { Compared }\end{array}$ & \multicolumn{6}{|c|}{ Number present in top 100 enriched proteins } \\
\hline WT vs. $\triangle$ SNAG & 7 & 4 & 2 & 0 & 2 & 0 \\
\hline WT vs. P2A & 8 & 5 & 3 & 0 & 2 & 0 \\
\hline SNAG vs. BirA* & 5 & 3 & 2 & 0 & 1 & 0 \\
\hline
\end{tabular}

Table 1. LSD1-dependent components of GFI1B proximitomes. Shown are components of LSD1-containing transcription complexes represented in the GFI1B proximitome. The core and unique components of each complex are enumerated (top), and the number of core and unique components from these complexes found in each proximitome comparison is shown. 
TABLE S1 Comparison of the top 100 LSD1-dependent protein candidates associated with GFI1B

\begin{tabular}{|c|c|c|c|c|c|c|}
\hline \multirow[b]{2}{*}{ Rank } & \multicolumn{2}{|c|}{ GFI1B-WT vs. GFI1B- $\Delta$ SNAG } & \multicolumn{2}{|c|}{ GFI1B-WT vs. GFI1B-P2A } & \multicolumn{2}{|c|}{ SNAG vs. BirA* Only } \\
\hline & Gene name & $P$ value $^{a}$ & Gene name & $P$ value ${ }^{a}$ & Gene name & $P$ value $^{a}$ \\
\hline 1 & FERMT3 & $6.13 E-22$ & ZNF451 & $3.22 \mathrm{E}-17$ & LSG1 & $9.06 \mathrm{E}-30$ \\
\hline 2 & PRDM10 & $2.22 \mathrm{E}-15$ & RCOR3 & $4.40 \mathrm{E}-16$ & FAM83H & $1.89 \mathrm{E}-26$ \\
\hline 3 & ZNF451 & $1.34 \mathrm{E}-13$ & GSE1 & $5.28 \mathrm{E}-14$ & MARCKSL1 & $5.14 \mathrm{E}-13$ \\
\hline 4 & RCOR3 & $2.28 \mathrm{E}-12$ & L3MBTL3 & $1.62 \mathrm{E}-13$ & PHF21A & $6.01 \mathrm{E}-21$ \\
\hline 5 & RCOR1 & $4.44 \mathrm{E}-12$ & RREB1 & $1.00 \mathrm{E}-12$ & RCOR3 & $7.48 \mathrm{E}-20$ \\
\hline 6 & KDM1A (LSD1) & $1.60 \mathrm{E}-11$ & ZNF622 & $1.98 \mathrm{E}-12$ & RREB1 & $2.20 \mathrm{E}-17$ \\
\hline 7 & GSE1 & $6.06 \mathrm{E}-11$ & CUL4B & $2.51 \mathrm{E}-12$ & KDM1A (LSD1) & $3.68 \mathrm{E}-16$ \\
\hline 8 & HMG20B & $1.42 \mathrm{E}-10$ & PRDM2 & $2.84 \mathrm{E}-12$ & PRKCA & 8.17E-16 \\
\hline 9 & RREB1 & $1.84 \mathrm{E}-10$ & CBX4 & $1.35 \mathrm{E}-11$ & AFTPH & $1.70 \mathrm{E}-14$ \\
\hline 10 & CBX4 & $2.37 \mathrm{E}-10$ & KDM1A (LSD1) & $6.68 \mathrm{E}-11$ & RCOR1 & $1.12 \mathrm{E}-13$ \\
\hline 11 & RAD54L2 & $3.46 \mathrm{E}-10$ & LARP1 & $7.48 \mathrm{E}-11$ & FASTKD1 & $5.37 \mathrm{E}-13$ \\
\hline 12 & L3MBTL3 & $3.51 \mathrm{E}-10$ & GFM1 & $1.53 \mathrm{E}-10$ & AP3B1 & 7.61E-13 \\
\hline 13 & PTK2B & $5.27 \mathrm{E}-10$ & SLX4 & $3.00 \mathrm{E}-10$ & NAP1L4 & $9.14 \mathrm{E}-13$ \\
\hline 14 & $\mathrm{P} 4 \mathrm{HB}$ & $1.44 \mathrm{E}-09$ & SEPT7 & $3.73 \mathrm{E}-10$ & NAP1L1 & $2.89 \mathrm{E}-12$ \\
\hline 15 & NUP188 & $1.11 \mathrm{E}-08$ & ZMYM2 & $6.66 \mathrm{E}-10$ & DNAJB12 & $4.40 \mathrm{E}-12$ \\
\hline 16 & EIF2B5 & $1.73 E-08$ & GIGYF2 & 1.13E-09 & TSR1 & 7.75E-12 \\
\hline 17 & HIST1H1A & $2.22 \mathrm{E}-08$ & SEC23B & 3.10E-09 & TMED9 & $2.09 \mathrm{E}-11$ \\
\hline 18 & FAM83H & 2.99E-08 & ARVCF & 3.85E-09 & ROR2 & $2.61 \mathrm{E}-11$ \\
\hline 19 & ZNF629 & $6.54 \mathrm{E}-08$ & RCOR1 & 4.77E-09 & MAP2K7 & $1.12 \mathrm{E}-10$ \\
\hline 20 & OSBPL3 & $9.68 \mathrm{E}-08$ & PRDM10 & 5.43E-09 & IPO9 & 1.33E-09 \\
\hline 21 & ARVCF & $1.45 \mathrm{E}-07$ & HMG20B & $6.28 \mathrm{E}-09$ & NOL11 & $1.08 \mathrm{E}-08$ \\
\hline 22 & HDAC2 & $1.58 \mathrm{E}-07$ & YARS & $1.03 \mathrm{E}-08$ & HMG20B & $1.51 \mathrm{E}-08$ \\
\hline 23 & PRDM2 & 1.77E-07 & PHF21A & $1.13 E-08$ & WDR43 & $2.01 \mathrm{E}-08$ \\
\hline 24 & ZMYM2 & 2.97E-07 & FAM208B & $1.20 \mathrm{E}-08$ & PPP4R3A & $4.59 \mathrm{E}-08$ \\
\hline 25 & BUB1B & $5.45 \mathrm{E}-07$ & ZNF609 & $1.78 \mathrm{E}-08$ & UBE2O & 8.66E-08 \\
\hline 26 & HDAC1 & 5.53E-07 & cDNA FLJ51764 & 4.26E-08 & RRP7BP & $1.12 \mathrm{E}-07$ \\
\hline 27 & NCKAP1 & $6.44 \mathrm{E}-07$ & MED14 & $6.05 \mathrm{E}-08$ & CHTF18 & $1.30 \mathrm{E}-07$ \\
\hline 28 & TARS2 & $6.78 \mathrm{E}-07$ & MAGEC1 & 7.59E-08 & L3MBTL3 & $1.45 \mathrm{E}-07$ \\
\hline 29 & HMG20A & $1.28 \mathrm{E}-06$ & RAB3GAP1 & $1.39 \mathrm{E}-07$ & $\mathrm{MTCH} 2$ & 2.66E-07 \\
\hline 30 & EDC4 & $1.80 \mathrm{E}-06$ & NASP & $3.22 \mathrm{E}-07$ & IPO7 & 4.73E-07 \\
\hline 31 & CBSL & $1.88 \mathrm{E}-06$ & BRD8 & $9.26 \mathrm{E}-07$ & TNRC18 & 4.94E-07 \\
\hline 32 & NCOA1 & $2.34 \mathrm{E}-06$ & RAI1 & $9.74 \mathrm{E}-07$ & DDX27 & 8.64E-07 \\
\hline 33 & KDM5B & 6.37E-06 & PIAS2 & $1.60 \mathrm{E}-06$ & NOP14 & $1.01 \mathrm{E}-06$ \\
\hline 34 & EHD4 & $6.58 \mathrm{E}-06$ & RSF1 & $2.66 \mathrm{E}-06$ & NOB1 & 1.61E-06 \\
\hline 35 & ZFPM1 & 8.87E-06 & GPATCH1 & $7.60 \mathrm{E}-06$ & BYSL & $1.62 \mathrm{E}-06$ \\
\hline 36 & SMARCAL1 & 1.26E-05 & NUP58 & 8.82E-06 & UBE3C & 1.83E-06 \\
\hline
\end{tabular}




\begin{tabular}{|c|c|c|c|c|c|c|}
\hline 37 & PHF21A & 1.53E-05 & KIF22 & 1.06E-05 & GSE1 & 3.06E-06 \\
\hline 38 & SEC23B & 1.75E-05 & RUNX1 & 1.13E-05 & PDAP1 & $4.41 \mathrm{E}-06$ \\
\hline 39 & ATRX & 3.32E-05 & HMG20A & 1.31E-05 & RRP1B & 4.42E-06 \\
\hline 40 & PEX1 & 4.92E-05 & IQSEC1 & $1.64 \mathrm{E}-05$ & RPL15 & $5.68 \mathrm{E}-06$ \\
\hline 41 & GRAP2 & $5.12 \mathrm{E}-05$ & MTF2 & $1.65 \mathrm{E}-05$ & PDCD11 & $1.09 \mathrm{E}-05$ \\
\hline 42 & ABCB10 & $6.04 \mathrm{E}-05$ & HDAC3 & $2.74 \mathrm{E}-05$ & BUD13 & $1.40 \mathrm{E}-05$ \\
\hline 43 & $\mathrm{HBZ}$ & $6.73 E-05$ & HDAC1 & 3.06E-05 & MRPS27 & 1.59E-05 \\
\hline 44 & AMBRA1 & 6.91E-05 & TRIM41 & $3.10 \mathrm{E}-05$ & RRBP1 & $1.62 \mathrm{E}-05$ \\
\hline 45 & ANKHD1 & $9.12 \mathrm{E}-05$ & SETD2 & $3.70 \mathrm{E}-05$ & MARS & 1.87E-05 \\
\hline 46 & STAT5B & 1.03E-04 & TUBB1 & 4.52E-05 & PRDM2 & $1.92 \mathrm{E}-05$ \\
\hline 47 & JMJD1C & 1.18E-04 & PTK2B & 4.54E-05 & IGF2BP2 & $3.99 \mathrm{E}-05$ \\
\hline 48 & CHD9 & 1.57E-04 & WRN & 6.36E-05 & PGM2 & 5.05E-05 \\
\hline 49 & SLX4 & $2.72 \mathrm{E}-04$ & ATRX & $8.12 \mathrm{E}-05$ & FDPS & $6.59 \mathrm{E}-05$ \\
\hline 50 & MAP1B & $2.73 E-04$ & PIAS1 & 1.17E-04 & OPA1 & 7.06E-05 \\
\hline 51 & PRR12 & 3.06E-04 & KPNA5 & 1.33E-04 & MYBBP1A & 7.87E-05 \\
\hline 52 & NCOR2 & $3.12 \mathrm{E}-04$ & ABCB10 & 1.69E-04 & GFI1B & $8.21 \mathrm{E}-05$ \\
\hline 53 & BCOR & $3.84 \mathrm{E}-04$ & NCOR2 & $1.80 \mathrm{E}-04$ & FBRSL1 & $9.39 \mathrm{E}-05$ \\
\hline 54 & SLC25A12 & 4.31E-04 & RAD18 & 1.91E-04 & CARM1 & $1.65 \mathrm{E}-04$ \\
\hline 55 & ISYNA1 & 5.51E-04 & ZMYND8 & 1.96E-04 & HMG20A & $1.70 \mathrm{E}-04$ \\
\hline 56 & XRCC5 & $9.24 \mathrm{E}-04$ & ZNF384 & $2.35 \mathrm{E}-04$ & NEPRO & $1.72 \mathrm{E}-04$ \\
\hline 57 & PYGB & 1.14E-03 & PIGT & $2.49 \mathrm{E}-04$ & COQ5 & 1.97E-04 \\
\hline 58 & FLII & 1.29E-03 & BCOR & $2.59 \mathrm{E}-04$ & LENG8 & $2.05 E-04$ \\
\hline 59 & TBL1X & 1.41E-03 & ZFPM1 & 3.06E-04 & ATN1 & $2.65 E-04$ \\
\hline 60 & OTUD4 & 1.46E-03 & TRNAU1AP & 3.35E-04 & COMMD3 & 3.37E-04 \\
\hline 61 & ABCD3 & $1.52 E-03$ & $\mathrm{NR} 2 \mathrm{C} 2$ & $3.85 \mathrm{E}-04$ & LTV1 & 3.46E-04 \\
\hline 62 & VPS52 & $1.62 \mathrm{E}-03$ & CKAP5 & 3.91E-04 & RRP12 & 4.01E-04 \\
\hline 63 & PIAS1 & 1.71E-03 & SMARCAL1 & $3.95 E-04$ & ORC1 & 4.56E-04 \\
\hline 64 & LARP4B & 1.79E-03 & CTR9 & $4.23 \mathrm{E}-04$ & FERMT3 & 4.92E-04 \\
\hline 65 & GALK2 & 1.98E-03 & FUBP3 & 4.51E-04 & PINX1 & $5.42 \mathrm{E}-04$ \\
\hline 66 & RUNX1 & $2.08 \mathrm{E}-03$ & cDNA FLJ51028 & $4.60 \mathrm{E}-04$ & GTF2H2C & 6.37E-04 \\
\hline 67 & UBAP2 & $2.13 \mathrm{E}-03$ & CNOT1 & $6.62 \mathrm{E}-04$ & ZNF701 & 7.84E-04 \\
\hline 68 & $\mathrm{FECH}$ & $2.21 \mathrm{E}-03$ & SENP6 & $6.64 \mathrm{E}-04$ & NUDT4 & 8.94E-04 \\
\hline 69 & PRRC2C & $2.35 \mathrm{E}-03$ & TAF6L & 7.46E-04 & KDM5B & $9.43 \mathrm{E}-04$ \\
\hline 70 & EXOC3L2 & $2.86 \mathrm{E}-03$ & ZNF592 & 7.49E-04 & RBFOX1 & 1.13E-03 \\
\hline 71 & ASNS & $2.88 \mathrm{E}-03$ & VPS51 & 7.89E-04 & SLC3A2 & $1.25 \mathrm{E}-03$ \\
\hline 72 & AHDC1 & 2.92E-03 & HBZ & 8.00E-04 & RPP40 & $1.28 \mathrm{E}-03$ \\
\hline 73 & CARD6 & 2.97E-03 & CBSL & 8.05E-04 & LIG3 & $1.42 \mathrm{E}-03$ \\
\hline 74 & RICTOR & $3.02 \mathrm{E}-03$ & PLCD1 & 9.81E-04 & CCDC9 & $1.78 \mathrm{E}-03$ \\
\hline 75 & YARS & 3.04E-03 & SDHA & 1.19E-03 & SLC2A1 & 1.99E-03 \\
\hline 76 & FHOD1 & 3.36E-03 & KDM5B & $1.21 \mathrm{E}-03$ & NOL6 & $2.24 \mathrm{E}-03$ \\
\hline
\end{tabular}




\begin{tabular}{|c|c|c|c|c|c|c|}
\hline 77 & ZNF672 & 4.07E-03 & SBNO1 & 1.48E-03 & TBC1D14 & 2.27E-03 \\
\hline 78 & C16orf58 & 4.26E-03 & TBL1X & $1.56 \mathrm{E}-03$ & HNRNPH3 & $2.59 \mathrm{E}-03$ \\
\hline 79 & HNRNPL & 4.49E-03 & ATXN2 & $1.75 \mathrm{E}-03$ & IMP4 & 2.71E-03 \\
\hline 80 & ALDH18A1 & $6.11 \mathrm{E}-03$ & TBL1XR1 & $1.79 \mathrm{E}-03$ & HIST1H1C & $2.99 \mathrm{E}-03$ \\
\hline 81 & GOLGA2 & $6.18 \mathrm{E}-03$ & RPA1 & $1.81 \mathrm{E}-03$ & MMS19 & $3.08 \mathrm{E}-03$ \\
\hline 82 & PLCD1 & 6.31E-03 & KIF5A & 1.87E-03 & NCOR2 & $3.19 \mathrm{E}-03$ \\
\hline 83 & TXNIP & 7.04E-03 & DGCR14 & $2.10 \mathrm{E}-03$ & RPL7L1 & 3.39E-03 \\
\hline 84 & ALDH16A1 & 7.11E-03 & TRMT2A & $2.13 \mathrm{E}-03$ & BRMS1 & $3.41 \mathrm{E}-03$ \\
\hline 85 & ATXN2 & 7.45E-03 & ACACA & 2.77E-03 & DDX56 & 3.77E-03 \\
\hline 86 & PSMB5 & 7.79E-03 & EXOC2 & $2.96 \mathrm{E}-03$ & KIF18A & $3.79 \mathrm{E}-03$ \\
\hline 87 & GFM1 & 8.53E-03 & $\mathrm{MSH} 3$ & 3.04E-03 & BAZ1B & 4.47E-03 \\
\hline 88 & CNOT2 & 1.07E-02 & NKTR & $3.20 \mathrm{E}-03$ & CSPP1 & $4.54 \mathrm{E}-03$ \\
\hline 89 & QSER1 & 1.07E-02 & HAUS5 & $3.50 \mathrm{E}-03$ & CBFB & 4.57E-03 \\
\hline 90 & DYRK1A & $1.09 \mathrm{E}-02$ & DDX51 & 3.79E-03 & SNAP23 & 4.96E-03 \\
\hline 91 & RANBP9 & 1.14E-02 & CHD9 & 4.34E-03 & $\mathrm{H} 2 \mathrm{AFV}$ & $5.23 E-03$ \\
\hline 92 & PPA2 & $1.18 \mathrm{E}-02$ & RNPC3 & 4.60E-03 & BRAF & $5.27 \mathrm{E}-03$ \\
\hline 93 & QARS & 1.19E-02 & ZNF512B & 5.33E-03 & IQSEC1 & 5.37E-03 \\
\hline 94 & BIRC2 & $1.23 E-02$ & SMG7 & $5.40 \mathrm{E}-03$ & ARAF & 5.77E-03 \\
\hline 95 & SMG7 & $1.25 \mathrm{E}-02$ & BEND3 & 5.92E-03 & EBNA1BP2 & $5.85 \mathrm{E}-03$ \\
\hline 96 & CD3EAP & $1.27 \mathrm{E}-02$ & ZKSCAN4 & $6.08 \mathrm{E}-03$ & USP36 & $6.04 \mathrm{E}-03$ \\
\hline 97 & MTFR1L & 1.36E-02 & RCOR3 & $6.49 \mathrm{E}-03$ & PRR5 & $6.15 \mathrm{E}-03$ \\
\hline 98 & UTP14C & $1.41 \mathrm{E}-02$ & HSD17B10 & $6.54 \mathrm{E}-03$ & PHIP & $6.71 \mathrm{E}-03$ \\
\hline 99 & KDM2B & 1.43E-02 & HUWE1 & $6.59 E-03$ & GTPBP4 & 6.94E-03 \\
\hline 100 & MAGEC1 & $1.50 \mathrm{E}-02$ & ALDH18A1 & $6.65 \mathrm{E}-03$ & MMTAG2 & 7.01E-03 \\
\hline
\end{tabular}

${ }^{a}$ Determined by two tailed $t$-distribution of the $z$-score of the 20 neighboring ranked proteins.

Known BHC complex members in RED 
Table S2 Oligonucleotides used in this study

\begin{tabular}{|c|c|c|}
\hline Oligo Name & Sequence ( $5^{\prime}$ to $\left.3^{\prime}\right)$ & Purpose \\
\hline hHMG20A qPCR Fwd & GATCTGGCTACCACTGGGTT & RT-qPCR \\
\hline hHMG20A qPCR Rev & СCTCCTCGTTTACTTCGTTGC & RT-qPCR \\
\hline hHMG20B qPCR2 Fwd & CATGGGGGCTTCGTGGTGA & RT-qPCR \\
\hline hHMG20B qPCR2 Rev & CGCCCAGCATCTTGGTGAT & RT-qPCR \\
\hline hGSE1 qPCR 1 Fwd & GCCTGCAATGCACTGGCCTA & RT-qPCR \\
\hline hGSE1 qPCR 1 Rev & GGAGCCTGGAATCCTTTGCTTC & RT-qPCR \\
\hline hPHF21A qPCR Fwd & СTCCTGCTGCTCTGATTCCC & RT-qPCR \\
\hline hPHF21A qPCR Rev & GGCCTCTCGCACTTTTTAGA & RT-qPCR \\
\hline hHBA1/2 qPCR Fwd & CCGGTCAACTTCAAGCTCCTA & RT-qPCR \\
\hline hHBA1/2 qPCR Rev & GGTATTTGGAGGTCAGCACGG & RT-qPCR \\
\hline hHBZ qPCR Fwd & GAGCAGGCCCAACTCCAG & RT-qPCR \\
\hline hHBZ qPCR Rev & TGTCGTCGATGCTCTTCACC & RT-qPCR \\
\hline hAHSP qPCR Fwd & ATAAGGATCTCATTTCCGCAGG & RT-qPCR \\
\hline hAHSP qPCR Rev & CCTTGTCTCGCTCTTGGGG & RT-qPCR \\
\hline hHBB qPCR Fwd & GTGAACGTGGATGAAGTTGGTGGTGAG & RT-qPCR \\
\hline hHBB qPCR Rev & TTGGACAGCAAGAAAGCGAGCTTAGTG & RT-qPCR \\
\hline hHBD qPCR Fwd & GCACGTGGATCCTGAGAACT & RT-qPCR \\
\hline hHBD qPCR Rev & ACCCTTGAAGTAGGCATTGTGT & RT-qPCR \\
\hline hHBE1 qPCR Fwd & GGCCAGAACTTCGGCAGTAA & RT-qPCR \\
\hline hHBE1 qPCR Rev & AAACAACGAGGAGTCTGCCC & RT-qPCR \\
\hline hHBG1/2 qPCR Fwd & ACTCGCTTCTGGAACGTCTG & RT-qPCR \\
\hline hHBG1/2 qPCR Rev & CCTCTGGGTCCATGGGTAGA & RT-qPCR \\
\hline hALAS2 qPCR Fwd & TACCCACCTGTCATTCGTTCG & RT-qPCR \\
\hline hALAS2 qPCR Rev & GGACCGGAAAATGGCTTCCT & RT-qPCR \\
\hline hFECH qPCR Fwd & ATCCAGCAGCTGGAGGGTCT & RT-qPCR \\
\hline hFECH qPCR Rev & TGAATCTTGGGGGTTCGGCG & RT-qPCR \\
\hline hGYPA qPCR Fwd & CATTAAGTACCACTGAGGTGGC & RT-qPCR \\
\hline hGYPA qPCR Rev & TGATGGGCAAGTTGTACCCT & RT-qPCR \\
\hline hKLF1 qPCR Fwd & TGACTTCCTCAAGTGGTGGC & RT-qPCR \\
\hline hKLF1 qPCR Rev & GGTGAGGAGGAGATCCAGGT & RT-qPCR \\
\hline hGFI1B qPCR Fwd & GCAGGAAGATGAACCGCTCT & RT-qPCR \\
\hline hGFI1B qPCR Rev & CCAGGCACTGGTTTGGGAA & RT-qPCR \\
\hline hMYB qPCR Fwd & GGGCAGAAATCGCAAAGCTACT & RT-qPCR \\
\hline hMYB qPCR Rev & GTTAACAGTGGGCTGGCCAG & RT-qPCR \\
\hline hCDKN1A qPCR Fwd & TTTCTCTCGGCTCCCCATGT & RT-qPCR \\
\hline \multirow[t]{2}{*}{ hCDKN1A qPCR Rev } & GCTGTATATTCAGCATTGTGGG & RT-qPCR \\
\hline & $\begin{array}{l}\text { CTAGCAAGGCAAATCTCATAGGCAAGTAC } \\
\text { CCTTTTTTTG }\end{array}$ & shRNA Knockdown (EZ Tet pLKO) \\
\hline
\end{tabular}


bioRxiv preprint doi: https://doi.org/10.1101/519090; this version posted January 13,2019 . The copyright holder for this preprint (which was not certified by peer review) is the author/funder. All rights reserved. No reuse allowed without permission.

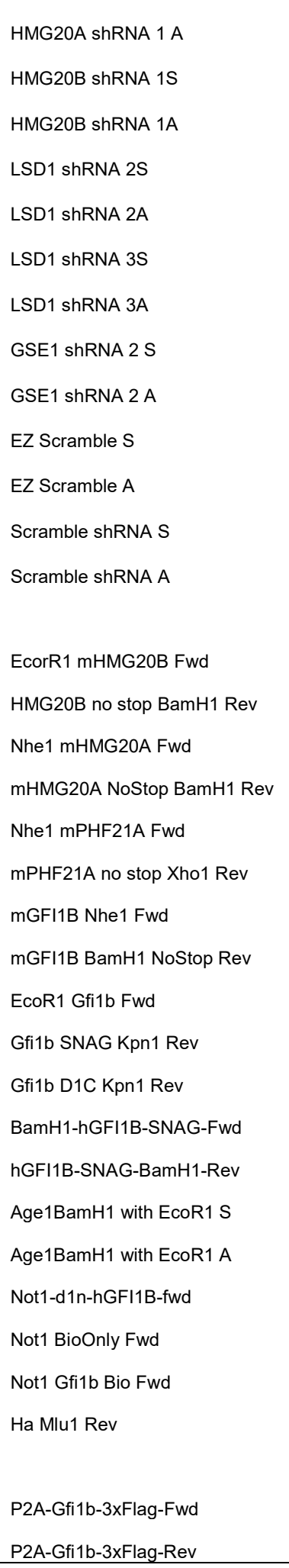

AATTCAAAAAAAGGCAAATCTCATAGGCAAGACTAGTACTTGCCTATGAG ATTTGCCTTG CCGGAGCATCCCTTTAGCTTTCAATCTCGAGATTGAAAGCTAAAGGGATG CTTTTTT

AATTAAAAAGCATCCCTTTAGCTTTCAATCTCGAGATTGAAAGCTAAAG GGATGCT

CCGGGCCTAGACATTAAACTGAATACTCGAGTATTCAGTTTAATGTCTAG GCTTTTT

AATTAAAAAGCCTAGACATTAAACTGAATACTCGAGTATTCAGTTTAATG TCTAGGC

CCGGGCTACATCTTACCTTAGTCATCTCGAGATGACTAAGGTAAGATGTA

GCTTTTT

AATTAAAAAGCTACATCTTACCTTAGTCATCTCGAGATGACTAAGGTAAG ATGTAGC

CTAGCGAACTCACCTTGACGTCAATGTACTAGTCATTGACGTCAAGGTGA GTTCTTTTTG

AATTCAAAAAGAACTCACCTTGACGTCAATGACTAGTACATTGACGTCAA GGTGAGTTCG

CTAGCCAACAAGATGAAGAGCACCAATACTAGTTTGGTGCTCTTCATCTT GTTGTTTTTG

AATTCAAAAACAACAAGATGAAGAGCACCAAACTAGTATTGGTGCTCTTC ATCTTGTTGG

CCGGCAACAAGATGAAGAGCACCAACTCGAGTTGGTGCTCTTCATCTTGT TGTTTTT

AATTAAAAACAACAAGATGAAGAGCACCAACTCGAGTTGGTGCTCTTCAT CTTGTTG

\author{
shRNA Knockdown (EZ Tet pLKO) \\ shRNA Knockdown (Tet pLKO) \\ shRNA Knockdown (Tet pLKO) \\ shRNA Knockdown (Tet pLKO) \\ shRNA Knockdown (Tet pLKO) \\ shRNA Knockdown (Tet pLKO) \\ shRNA Knockdown (Tet pLKO) \\ shRNA Knockdown (EZ Tet pLKO) \\ shRNA Knockdown (EZ Tet pLKO) \\ shRNA Knockdown (EZ Tet pLKO) \\ shRNA Knockdown (EZ Tet pLKO) \\ shRNA Knockdown (Tet pLKO) \\ shRNA Knockdown (Tet pLKO)
}

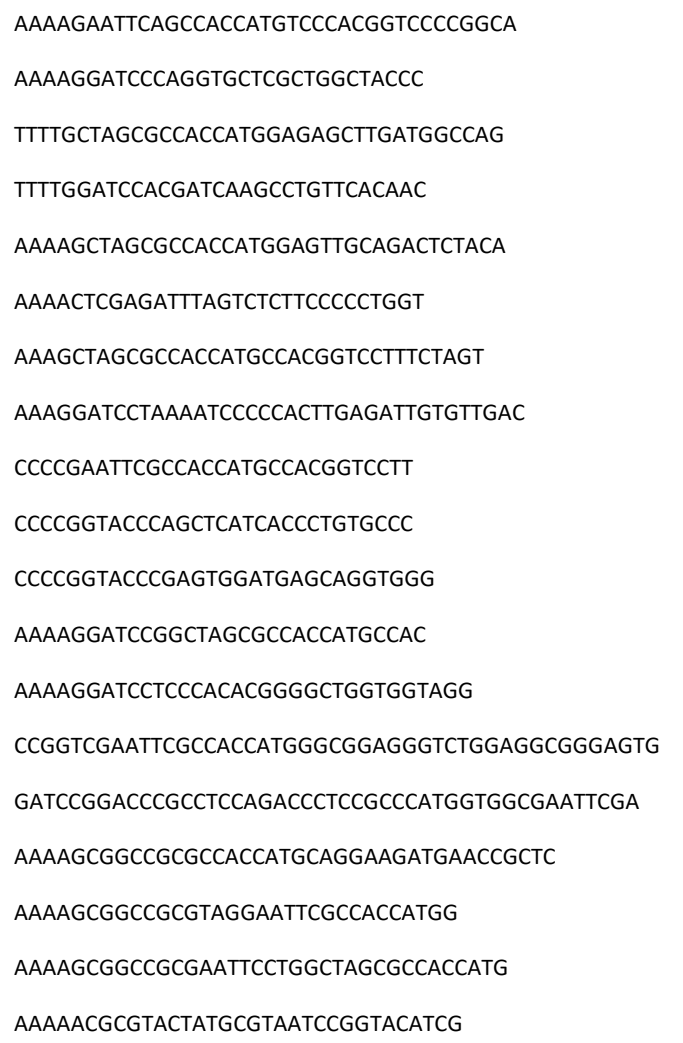

Cloning HMG20B into pcDNA3.1

Cloning HMG20B into pcDNA3.1

Cloning HMG20A into pcDNA3.1

Cloning HMG20A into pcDNA3.1

Cloning PHF21A into pcDNA3.1

Cloning PHF21A into pcDNA3.1

Cloning GFI1B into pcDNA3.1

Cloning GFI1B into pcDNA3.1

Cloning GFI1B forms inframe with Gal4

Cloning GFI1B forms inframe with Gal4

Cloning GFI1B forms inframe with Gal4

Cloning SNAG-BirA*:HA (pcDNA3.1)

Cloning SNAG-BirA*:HA (pcDNA3.1)

Cloning BirA*:HA Only (pcDNA3.1)

Cloning of BirA*:HA Only (pcDNA3.1)

Cloning dSNAG-BirA*:HA (pLVX-Tight)

Cloning BirA*:HA only (pLVX-Tight)

Cloning GFI1B-BirA*:HA (pLVX-Tight)

Cloning GFI1B-BirA*:HA (pLVX-Tight) 